\title{
The Role of Sulfhydryl Reactivity of Small Molecules for the Activation of the KEAP1/NRF2 Pathway and the Heat Shock Response
}

\author{
Albena T. Dinkova-Kostova ${ }^{1,2}$ \\ ${ }^{1}$ Jacqui Wood Cancer Centre, Division of Cancer Research, Medical Research Institute, Ninewells Hospital and Medical School, \\ University of Dundee, James Arrott Drive, Dundee DD1 9SY, UK \\ ${ }^{2}$ Department of Pharmacology and Molecular Sciences and Department of Medicine, Johns Hopkins University School of Medicine, \\ Baltimore, MD 21205, USA \\ Correspondence should be addressed to Albena T. Dinkova-Kostova, a.dinkovakostova@dundee.ac.uk
}

Received 2 October 2012; Accepted 7 November 2012

Academic Editors: U. Hansen, H. Iwano, S. Parthasarathy, C. Riganti, and M. Shimojo

Copyright (C) 2012 Albena T. Dinkova-Kostova. This is an open access article distributed under the Creative Commons Attribution License, which permits unrestricted use, distribution, and reproduction in any medium, provided the original work is properly cited.

\begin{abstract}
The KEAP1/NRF2 pathway and the heat shock response are two essential cytoprotective mechanisms that allow adaptation and survival under conditions of oxidative, electrophilic, and thermal stress by regulating the expression of elaborate networks of genes with versatile protective functions. The two pathways are independently regulated by the transcription factor nuclear factorerythroid 2 p45-related factor 2 (NRF2) and heat shock factor 1 (HSF1), respectively. The activity of these transcriptional master regulators increases during conditions of stress and also upon encounter of small molecules (inducers), both naturally occurring as well as synthetically produced. Inducers have a common chemical property: the ability to react with sulfhydryl groups. The protein targets of such sulfhydryl-reactive compounds are equipped with highly reactive cysteine residues, which serve as sensors for inducers. The initial cysteine-sensed signal is further relayed to affect the expression of large networks of genes, which in turn can ultimately influence complex cell fate decisions such as life and death. The paper summarizes the multiple lines of experimental evidence demonstrating that the reactivity with sulfhydryl groups is a major determinant of the mechanism of action of small molecule dual activators of the KEAP1/NRF2 pathway and the heat shock response.
\end{abstract}

\section{Introduction}

One of the least abundant amino acids in all organisms, cysteine, has unique chemical features which nature uses in multifaceted ways. The chemical reactivity and plasticity of the sulfhydryl group of cysteine are in the heart of many molecular phenomena such as enzyme-based catalysis, metal coordination, protein folding, and protein-protein interactions. This versatility in turn affects some of the most fundamental biological processes such as cell division, proliferation, differentiation, apoptosis, cell signaling, and responses to oxidative, nitrosative, and electrophilic stress. In addition, $S$-thiolation, $S$-nitrosation, and the formation of disulfide bonds and sulfenic acid, or the so-called "reactive sulfur species" [1], that occur within cysteine-containing proteins can further affect the function of many other proteins that associate with the host molecule which harbours such modifications. Furthermore, the complex relationships between cysteine-containing redox-regulated proteins and their interacting networks, collectively termed as "the cellular thiolstat," have essential contributions to cell fate decisions [2]. It is therefore perhaps not surprising that sulfhydryl-reactive small molecules, both naturally occurring and synthetic, can profoundly alter a wide range of physiological processes by reacting with cysteine residues within their protein targets. This paper focuses on the importance of sulfhydryl reactivity of such small molecules for activation of two quintessential cytoprotective mechanisms: the KEAP1/NRF2 pathway and the heat shock response. 


\section{The KEAP1/NRF2 Pathway}

The KEAP1/NRF2 pathway, also known as "the phase 2 response" [3] or "the electrophile counterattack response" [4], is an essential cellular defense mechanism which regulates the expression of more than 500 genes [5]. Transcription factor nuclear factor-erythroid 2 p 45 -related factor 2 (NRF2), which operates as an obligatory heterodimer with a small MAF transcription factor, is the main orchestrator of this transcriptional program [6]. The predominant way of regulation of the levels of NRF2 within the cell is by control of its protein stability. At basal state, NRF2 is a very unstable protein with a half-life of just a few minutes [7] as it is continuously targeted for ubiquitination and proteasomal degradation by the repressor protein Kelch-like $\mathrm{ECH}$-associated protein 1 (KEAP1) [8], which functions as a substrate adaptor for Cullin-3- (Cul3-) based E3 ubiquitin ligase (Figure 1(a)) [9-11]. Recently, another pathway of KEAP1-independent regulation of NRF2 was uncovered, by the concerted action of glycogen synthase kinase- $3 \beta$ $(\mathrm{GSK} 3 \beta)$ and $\beta$-transducin repeat-containing protein $(\beta$ $\operatorname{TrCP}$ ) which serves as a substrate adaptor for Cullin-1(Cul1-) based E3 ubiquitin ligase [12-14]. Several different models have been proposed to explain the intricate mechanistic details of operation of the KEAP1/NRF2 pathway under basal as well as induced conditions, and these have been recently reviewed [15-17]. One common feature of these models is that, in addition to being a repressor and a substrate adaptor protein for the ubiquitination of NRF2, KEAP1 is also the sensor for inducers. This inducersensing capability of KEAP1 is due to its highly reactive cysteine residues which chemically interact with inducers of many different types and structures [18-21], leading to the loss of its substrate adaptor function. The loss of function of KEAP1 leads to stabilization of NRF2, nuclear accumulation of the transcription factor, and activation of the expression of genes that have antioxidant response elements (AREs), also known as electrophile-responsive elements (EpRE), in their promoter regions with the core consensus sequence $5^{\prime}$-TGABnnnGC- $3^{\prime}$ (where $\mathrm{B}=\mathrm{C}$, or $\mathrm{G}$, or T, and the letter " $\mathrm{n}$ " represents any nucleotide) [2229].

The ARE-containing NRF2-dependent genes encode a large network of proteins with versatile cytoprotective functions. The earliest recognized ARE-dependent genes were those which participate in the metabolism and transport of endo- and xenobiotics, such as glutathione $S$-transferases (GSTs), NAD(P)H:quinone oxidoreductase 1 (NQO1), epoxide hydrolase, and UDP glucuronosyltransferases [26-34]. Induction of these enzymes is also accompanied by elevation in glutathione levels [31], due to the ARE- and NRF2dependent transcriptional upregulation of both the heavy and the light subunits of the heterodimeric $\gamma$-glutamylcysteine ligase, the enzyme which catalyzes the rate-limiting step in the de novo biosynthesis of glutathione [35-37]. The identification of multiple functional AREs in the upstream regulatory regions of heme oxygenase 1 (HO-1) added this enzyme to the group of ARE-dependent gene products [38]. The use of differential hybridization methodology revealed that the upregulation of the already known ARE-dependent genes was also accompanied by the coordinate expression of genes encoding the heavy and light subunits of ferritin as well as anti-inflammatory enzymes such as leukotriene $\mathrm{B}_{4}$ dehydrogenase [39-41]. Global gene expression profiling confirmed previous findings and added new knowledge regarding the role of NRF2 in maintaining the cellular redox homeostasis and providing reducing equivalents. It became clear that several enzymes that participate in the synthesis, utilization, and regeneration of glutathione, thioredoxin, and $\mathrm{NAD}(\mathrm{P}) \mathrm{H}$ are also regulated by this transcription factor, including $\chi$-CT, the core subunit of the cystine/glutamate membrane transporter, $\gamma$-glutamylcysteine ligase catalytic and modulatory subunits, glutathione reductase, thioredoxin, thioredoxin reductase, glucose 6-phosphate dehydrogenase, 6-phosphogluconate dehydrogenase, and malic enzyme [42-44]. Interestingly, the expression of genes which code for proteins that participate in the repair and removal of damaged proteins, such as subunits of the $26 \mathrm{~S}$ proteasome, was also found to be NRF2 dependent [43]. Most recently, it was discovered that NRF2 contributes to the regulation of purine biosynthesis by controlling the expression of genes encoding enzymes in the pentose phosphate pathway $[45,46]$. By integrating chromatin immunoprecipitation with parallel sequencing (ChIP-Seq) and global transcription profiling, Malhotra et al. identified 645 basal and 654 inducible direct targets of NRF2, with 244 genes at the intersection [5]. Thus, the number and the functional diversity of the NRF2-dependent cytoprotective proteins is extraordinary and provides the cell with multiple defense mechanisms.

The generation of NRF2-knockout mice $[6,47]$ confirmed that NRF2 is the major orchestrator of the cellular stress response to oxidants and electrophiles. Indeed, NRF2knockout cells and animals are much more sensitive to the damaging effects of electrophiles, oxidants, and inflammatory agents in comparison to their wildtype counterparts; conversely, pharmacological or genetic activation of NRF2 has protective effects in numerous models of chronic disease, including cancer $[29,48,49]$. The failure of the NRF2knockout mice to adapt to conditions of stress results in greatly accelerated disease pathogenesis in comparison to wildtype animals $[50,51]$. However, the cytoprotective properties of the KEAP1/NRF2 pathway can be exploited by tumor cells to promote their survival. Indeed, NRF2 is frequently constitutively activated in established tumors. Mutations in KEAP1 or NRF2, which abrogate formation of the complex and lead to NRF2 accumulation and constitutive upregulation of the pathway, have been detected in several types of human cancer, including cancer of the lung, oesophagus, ovary, gallbladder, and skin [52-57]. A very recent comprehensive genomic characterisation study on squamous cell lung cancer conducted by the Cancer Genome Atlas Research Network reported the occurrence of mutations in NRF2, KEAP1, or CUL3 in $34 \%$ of 178 lung squamous cell carcinomas [58]. In addition, under conditions of oncogenic stress, such as that occurring during permanent activation of oncogenic K-RAS and B-RAF, activation of NRF2 can facilitate cell proliferation [59]. 


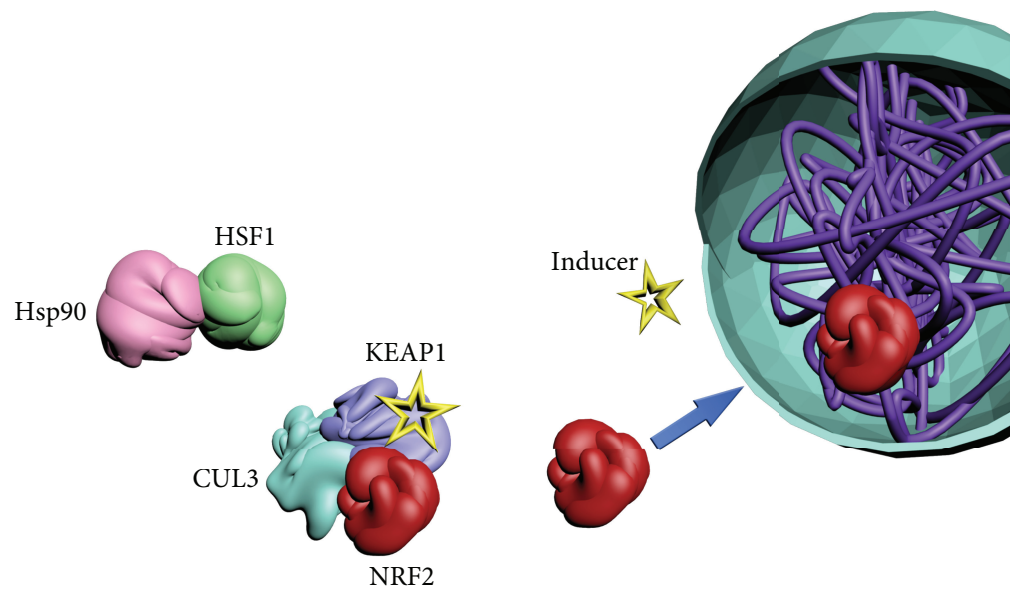

(a)

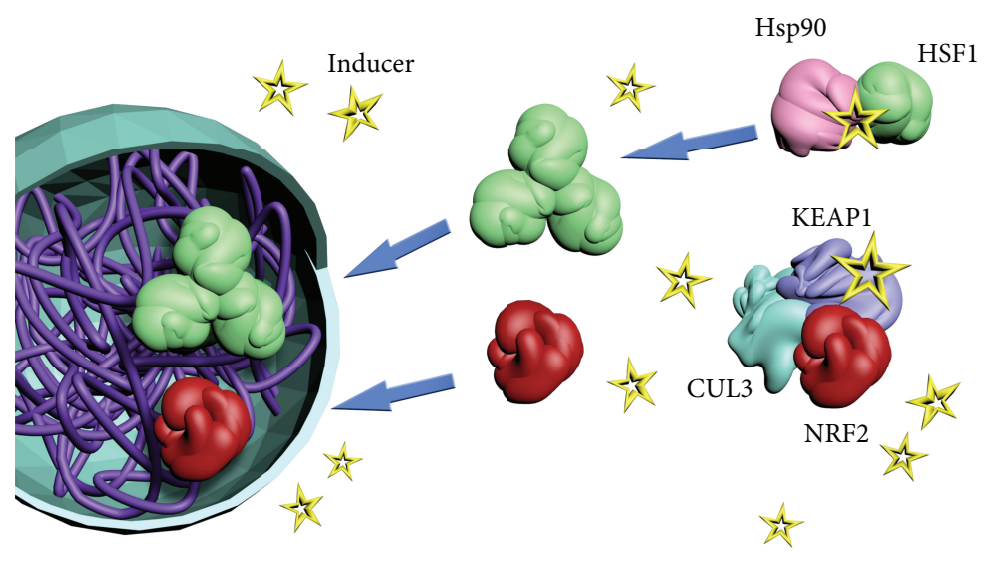

(b)

FIGURE 1: The KEAP1/NRF2 pathway and the heat shock response are highly inducible essential defense systems that allow the cell to adapt and survive under various conditions of stress by regulating the expression of elaborate networks of several hundred genes with versatile cytoprotective functions. At low concentrations (a), inducers (yellow stars) bind to cysteine residues of the protein sensor KEAP1 (purple). Consequently, KEAP1 loses its ability to target transcription factor NRF2 (red) for ubiquitination via CUL3 (cyan)-dependent E3 ubiquitin ligase, preventing subsequent proteosomal degradation of NRF2 and allowing its accumulation, nuclear translocation, and binding to the ARE as a heterodimer with a small MAF protein (not shown) to induce expression of NRF2-target genes. At high concentrations (b), in addition to activating the KEAP1/NRF2 pathway, inducers also activate the heat shock response by chemically reacting with transcription factor HSF1 (green), or a negative regulator of HSF1, such as Hsp90 (pink) or Hsp70 (not shown). As a result, HSF1 dissociates from the protein complex, trimerizes, undergoes complex posttranslational modifications, translocates to the nucleus, and induces expression of HSF1-target genes.

It was also recently shown that when PI3 K-AKT signalling is sustained, NRF2 redirects glucose and glutamine into anabolic pathways, and thus affects primary metabolism [45]. Such conditions have the potential to favor tumor development and, coupled with the increased activity of detoxification enzymes, to also decrease therapeutic efficacy and contribute to drug resistance. This double-edged nature of the consequences of either downregulating or upregulating the KEAP1/NRF2 pathway poses a major challenge for the development and implementation of inducers or inhibitors of this pathway as chemopreventive and/or chemotherapeutic agents.

\section{The Heat Shock Response}

Another essential inducible defense mechanism is the heat shock response which protects the cell under conditions of acute and chronic proteotoxic stress and preserves the integrity of the proteome. The central regulator of the heat shock response is transcription factor heat shock factor 1 (HSF1) [60, 61]. Under basal conditions, HSF1 is an inactive monomeric phosphoprotein bound to heat shock protein 90 (Hsp90) (Figure 1(b)). Upon activation of the heat shock response by thermal stress or small-molecule inducers, HSF1 dissociates from the Hsp90 complex and forms a DNA 
binding-competent trimer which binds to heat shock elements (HSEs, consensus inverted repeat sequences nGAAn) in the regulatory regions of its target genes and activates their transcription [62, 63]. HSF1 undergoes a number of posttranslational modifications, such as phosphorylation, sumoylation, and acetylation, all of which have been implicated in regulating its transcriptional activity. This transcription factor is also negatively feedback regulated by the products of the genes whose expression it regulates, that is, heat shock proteins, such as Hsp70 and Hsp40.

By the use of differential display, transcriptional profiling, and proteomic approaches, it has been shown that, depending on the experimental system, the number of proteins whose gene expression is induced by heat shock ranges between 50 and 200 [64] and includes molecular chaperones that prevent unspecific aggregation of nonnative or partially misfolded proteins (e.g., Hsp70 and Hsp40), proteolytic proteins that participate in the removal of irreversibly damaged proteins (e.g., BAG3 (BCL2-associated protein), APG5L (protein involved in autophagy), CASP1 (caspase, cysteine protease), NEDD4L (neural precursor cell-expressed developmentally downregulated 4 like, ubiquitin-protein ligase)), RNA- and DNA-modifying enzymes, which participate in the repair of damaged DNA (e.g., the bacterial DNA glycosylase MutM), metabolic enzymes that maintain the energy supply of the cell [e.g., ACAT2 (acetyl-CoA acetyltransferase), ALAS1 (aminolevulinate synthase), ChGn (chondroitin $\beta-1,4-N$-acetylgalactosaminyltransferase], transcription factors, kinases, or phosphatases that further activate other stress response pathways [e.g., RHOH (Ras homolog), PTPG1 (tyrosine phosphatase), RGS2 (regulator of G-protein signaling), IER5 (regulator of immediate early response)], proteins involved in sustaining cellular structures such as the cytoskeleton and membranes (e.g., TJP4 (tight junction associated protein), SIPA1L3 (signal-induced proliferationassociated 1-like protein 3)), and proteins involved in transport and detoxification (e.g., the amino acid transporter SLC38A2).

Similar to the case of the KEAP1/NRF2 pathway, activation of HSF1 commonly occurs in cancer. Recent work has shown that, in malignant tumors, HSF1 is responsible for the orchestration of a transcriptional program, termed the HSF1 cancer program, which is distinct from the transcriptional response that is induced by heat shock. This program includes both HSF1 positively and negatively regulated genes and drives the expression of cancer-specific genes supporting oncogenic processes such as cell-cycle regulation, mitosis, energy metabolism, translation, cell signalling, and adhesion [65]. Moreover, this HSF1 cancer program was found to be very significantly and broadly associated with poor outcomes such as metastasis and death in breast cancer, whereby patient survival decreases dose dependently as HSF1 nuclear levels increase $[65,66]$. Further analysis of multiple independent gene expression data sets from patients with known clinical outcomes has revealed that activation of the HSF1 cancer program is also associated with reduced survival in colon and lung cancer, and, importantly, much more significantly than any individual, or even a panel of, HSP transcript(s) [65]. These findings demonstrate the very broad role of HSF1 which extends beyond the control of gene expression of heat shock proteins and the heat shock response and impacts cell survival in multiple ways.

\section{Reactivity with Sulfhydryl Groups: A Common Chemical Property of Small Molecule Dual Activators of the KEAP1/NRF2 Pathway and the Heat Shock Response}

The KEAP1/NRF2 pathway and the heat shock response are distinct cytoprotective mechanisms that are regulated by independent transcription factors which orchestrate specific transcriptional programs. Curiously, however, many small molecules which have been found to activate one of these pathways were independently shown to induce the other (Table 1). Examples include the endogenous 4-hydroxy-2-nonenal, nitro-fatty acids, 15deoxy- $\Delta^{12,14}$-prostaglandin $J_{2}$, acrolein, and hydrogen peroxide; the naturally occurring phytochemicals celastrol, sulforaphane, withaferin A, gedunin, and curumin; as well as the synthetic bis(benzylidene)alkanones, sulfoxythiocarbamates, oxidizable diphenols, and diamide. Although such compounds are able to participate in chemical reactions that are not limited to those with sulfhydryl groups, sulfhydryl reactivity (by oxidation-reduction, alkylation, or thiol-disulfide interchange) is their only common attribute. Seminal studies by Paul Talalay and his colleagues led to the recognition of this chemical property as a unifying feature of inducers of the phase 2 response [67]. It was then hypothesized that the protein sensor for inducers, which many years later was identified as KEAP1, must contain highly reactive cysteine residues. The development and use of a microtiterbased bioassay in the murine hepatoma Hepa1c1c7 cell line for screening of potential inducers of the NRF2-target gene NQO1 [68, 69] further strengthened the concept that sulfhydryl reactivity constitutes the only common property of both naturally occurring as well as synthetic inducers. NQO1 inducers were grouped in 10 distinct chemical classes: (i) Michael acceptors (olefins or acetylenes conjugated with electron-withdrawing groups), (ii) oxidizable diphenols and diamines, (iii) conjugated polyenes, (iv) hydroperoxides, (v) trivalent arsenicals, (vi) heavy metals, (vii) isothiocyanates, (viii) dithiocarbamates, (ix) dithiolethiones, and (x) vicinal dimercaptans [70].

In a large series of semisynthetic pentacyclic triterpenoids, the presence of the 1-en-3-one functional group (i.e., Michael acceptor) in the A-ring was found to be essential for inducer activity, and the introduction of a cyanoenone functionality, which strengthens the electrophilicity, further increased the inducer potency $[134,135]$. These experimental findings were also supported by molecular orbital calculations [136]. More recently, tricyclic and monocyclic cyanoenone derivatives were synthesized; some of which are extremely potent and remarkably bioavailable inducers in cell systems as well as in vivo in animals [19, 21, 137, 138]. Oxidizable diphenols (such as tBHQ and catechol estrogen metabolites) represent another class of inducers, 
TABLE 1: Examples of small molecules-dual activators of transcription factors NRF2 and HSF1.

\begin{tabular}{|c|c|c|}
\hline Compound name & Chemical structure & References \\
\hline 4-Hydroxy-2-nonenal & & {$[71-74]$} \\
\hline 10-Nitro-oleic acid & & {$[75,76]$} \\
\hline 15 -Deoxy- $\Delta^{12,14}$-prostaglandin $\mathrm{J}_{2}$ & & {$[73,74,76-79]$} \\
\hline Acrolein & & {$[71,80]$} \\
\hline $\mathrm{H}_{2} \mathrm{O}_{2}$ & & {$[4,81-83]$} \\
\hline Celastrol & & {$[73,84-87]$} \\
\hline Sulforaphane & & {$[76,78,88-91]$} \\
\hline Phenethyl isothiocyanate & & {$[92]$} \\
\hline Sulfoxythiocarbamate alkyl & & {$[86,93]$} \\
\hline Withaferin A & & {$[94,95]$} \\
\hline
\end{tabular}


Table 1: Continued.

\begin{tabular}{|c|c|c|}
\hline Compound name & Chemical structure & References \\
\hline Curcumin & & {$[96-108]$} \\
\hline Bis(2-hydroxy-benzylidene)acetone & & {$[86,109]$} \\
\hline Gedunin & & {$[94,110-113]$} \\
\hline Diamide & & {$[73,114]$} \\
\hline tBHQ (and related oxidizable diphenols) & & {$[20,71,76,78,81,88,115-119]$} \\
\hline Sodium arsenite & $\mathrm{O}=\mathrm{As}-\mathrm{O}^{-} \mathrm{Na}^{+}$ & {$[4,71,120-127]$} \\
\hline 1,2-Dithiole-3-thione & & {$[39-41,43,128]$} \\
\hline Heavy metals & $\mathrm{Cd}^{++}, \mathrm{Cu}^{++}$ & {$[4,71,73,76,129-133]$} \\
\hline
\end{tabular}

and activation of NRF2 by these compounds requires oxidation to their corresponding quinone derivatives [20, 139, 140], which then react with cysteine residues of KEAP1 $[20,71,81,88,141]$. In MCF-7 human breast cancer cells stably transfected with an ARE-luciferase reporter (AREc32 cells), tBQ and estradiol-3,4-quinone induce ARE-dependent gene expression [20]. In contrast, short-term (30 min) exposure to tBHQ, 2-hydroxyestradiol, 4-hydroxyestradiol, or 4-hydroxyestrone has a much more modest effect on the luciferase gene expression. Importantly, however, induction by these oxidizable diphenols is greatly potentiated (by $\sim 4$ 5 -fold) under conditions that favor formation of the corresponding quinones, that is, in the presence of transition metals and $\mathrm{O}_{2}$, strongly suggesting that the electrophilic quinone metabolites are the ultimate inducers. Trivalent arsenicals, such as phenylarsine oxide and sodium arsenite, react readily with vicinal sulfhydryl groups to form highly stable fivemembered cyclic products [142] and are potent NRF2 activators [4]. Two recent high-throughput screens, one employing an ARE-dependent luciferase reporter to screen 1.2 million compounds in human neuroblastoma IMR-32 cells [143] and another utilizing a luciferase reporting on the stabilization of NRF2 which evaluated 20,000 biologically active compounds in human neuroblastoma SH-SY5Y-Neh2-luc cells [110], have confirmed that sulfhydryl reactivity is a prominent feature of inducers of the KEAP1/NRF2 pathway. One of the most potent inducers identified in the ARE-reporter assay, a chloroquinolone derivative, has an electrophilic aryl chloride group located at the $\beta$-position of two $\alpha, \beta$-unsaturated carbonyl moieties, which could potentially be displaced 
by a nucleophilic sulfhydryl group through a 1,4-additionelimination reaction. Gedunin, a natural product identified as a new inducer in the NRF2-stabilization luciferase reporter assay has two electrophilic centers and, interestingly, had previously been identified as an activator of HSF1 and an inducer of Hsp70 [111].

Reactivity with sulfhydryl groups is also emerging as a common property of small molecule inducers of the heat shock response. Activation of HSF1 and enhanced transcription of Hsp70 have been observed upon exposure of cells to heavy metals such as $\mathrm{Cd}^{++}$and $\mathrm{Cu}^{++}$ [129-133], as well as alkylating agents such as iodoacetamide [144], the anticancer drugs 1,3-bis-(2-chloroethyl)1-nitrosourea, 1-(2-chloroethyl)-3-cyclohexyl-1-nitrosourea $[145,146]$, and nephrotoxic cysteine conjugates-derived reactive electrophilic metabolites [147]. The trivalent arsenical sodium arsenite activates HSF1 and induces heat shock proteins [120-127]. The isothiocyanates sulforaphane and phenethyl isothiocyanate induce heat shock proteins in cultured cells $[89,148]$ and in vivo in animals that had been administered these agents $[90,92]$. The double Michael acceptor-containing curcumin, the principal coloring and flavoring agent of curry, is also an inducer of heat shock proteins [96-102]. Hepatic murine Hsp70 and Hsp40 were induced $24 \mathrm{~h}$ after treatment with 1,2-dithiole-3-thione, and this induction was observed in livers of both wildtype as well as NRF2-knockout mice [128]. Curiously, pyrrolidine dithiocarbamate, which can react with sulfhydryl groups by thiol-interchange reactions, also activates $\operatorname{HSF} 1[149,150]$. The electrophilic oxidized and nitrated lipids such as 4hydroxy-2-nonenal, 15-deoxy- $\Delta^{12,14}$-prostaglandin $\mathrm{J}_{2}$, and 10-nitro-octadecenoic acid have all been shown to induce the HSF1-mediated heat shock response. Thus, exposure of human colon cancer cells to 4-hydroxy-2-nonenal caused nuclear accumulation of HSF1 and induced endogenous Hsp70 and Hsp40, as well as a luciferase reporter under the transcriptional control of the consensus HSE, and silencing of the expression of HSF1 by siRNA abolished this induction [72]. In a myocardial ischemia and reperfusion model in male Wistar rats, 15 -deoxy- $\Delta^{12,14}$-prostaglandin $J_{2}$ enhanced DNA-binding activity of HSF1 and induced the expression of Hsp70 [77]. Genome-wide transcriptional profiling of human endothelial cells after treatment with 10-nitrooctadecenoic acid revealed that the heat shock response is the major pathway activated by this nitro-fatty acid [75]. Similarly, induction of heat shock proteins, such as Hsp70 and Hsp40, was found to be a characteristic feature of the transcriptional response of A459 human lung cancer cells to the electrophilic $\alpha, \beta$-unsaturated aldehyde acrolein [80]. The quinone methide-containing natural product celastrol is a potent inducer of the heat shock response $[84,85]$, and this electrophilic moiety has been preserved during the design and synthesis of celastrol analogues as affinity probes for identification of its protein targets [151]. Other phytochemicals which have been shown to induce the heat shock response, such as sulforaphane, withaferin A, gedunin, and curcumin, also have the ability to react with sulfhydryl groups. Curiously, high concentrations of $17 \beta$-estradiol activate HSF1 and induce Hsp70 $[115,116]$, and it could be speculated that, similar to the activation of NRF2, the actual activators of HSF1 could be the electrophilic quinone derivatives of the catechol metabolites of 17 $\beta$-estradiol, 2-hydroxy and 4-hydroxyestradiol [152]. A high-throughput screen for HSF1 activators in which a library of more than 80,000 compounds was tested identified the presence of an $\alpha, \beta$-unsaturated carbonyl moiety as an essential structural requirement for induction of the heat shock response [94]. Among the active compounds were various natural products such as the limonoids, anthothecol, cedrelone, gedunin, and 7-desacetoxy-6,7-dehydrogedunin, the macrocyclic lactone dehydrocurvularin, and the steroidal lactone withaferin $\mathrm{A}$, as well as celastrol, all of which have an $\alpha, \beta$-unsaturated carbonyl functional group. A similar finding was made in an even larger scale study of approximately 1 million compounds, in which electrophilicity was found to be a common characteristic of the $\sim 200$ small molecule activators of the heat shock response that were identified [132].

To test the functional importance of sulfhydryl reactivity for activation of the KEAP1/NRF2 pathway and the heat shock response, inducers of these pathways have been used in combination with classical nucleophiles. One example is the quinone methide celastrol. Nucleophiles, such as cysteine and glutathione, participate in reversible regioselective and stereospecific addition reactions with celastrol to form Michael adducts, such that the nucleophilic attack is favored exclusively at the $\beta$-face with the nucleophile approach syn to the $\beta$-C9 methyl [153]. The ability of a biotinylated derivative of celastrol to bind to tubulin in cells is abolished in the presence of dithiothreitol (DTT), demonstrating the importance of the electrophilic moiety of celastrol for reaction with its intracellular protein targets [151]. In agreement, induction of both NRF2- and HSF1-dependant genes by celastrol are prevented by incubation with DTT or $\mathrm{N}$-acetylcysteine [84, 132], and the ability of the quinone methide to induce Hsp70, downregulate Cdk4 and Cyclin D1, and cause cell cycle arrest is reversed by $\mathrm{N}$-acetylcysteine and reduced glutathione, but not vitamin C or oxidized glutathione [154]. Similarly, activation of HSF1 by both diamide and $15 \mathrm{~d}-\mathrm{PGJ}_{2}$ is completely abolished by incubation of these compounds with a 5 -fold or greater excess of DTT prior to introducing them into the cell culture medium [73]. DTT also blocks the induction of an hsp70-luciferase reporter by sodium arsenite [120]. Whereas treatment with pyrrolidine dithiocarbamate caused dose- and time-dependent HSF activation, this activity is significantly inhibited by $N$-acetylcysteine and completely abolished by DTT [149]. The simultaneous addition of $N$-acetylcysteine and withaferin A to a reporter system in which the expression of enhanced green fluorescent protein is controlled by a minimal consensus HSE-containing promoter suppresses heat shock activation almost completely [94]. In the case of the NRF2 activation, elevating the intracellular glutathione levels by the inducer sulforaphane lowers, whereas depletion of glutathione by inhibiting its biosynthesis with buthionine sulfoximine increases the inducer potency of oxidizable diphenols [20].

Evaluation of structural analogues of small molecule activators of NRF2 and/or HSF1 which either lack the 


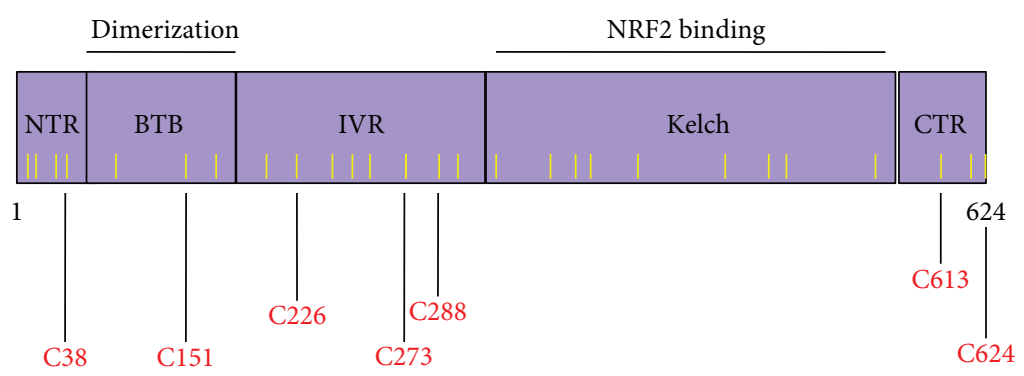

FIgURE 2: Domain structure of human KEAP1. NTR: N-terminal region (amino acids 1-60); BTB: broad complex, Tramtrack, Bric-á-brac (amino acids 61-179). KEAP1 forms a homodimer through the BTB binding domain; IVR: intervening region (amino acids 180-314); Kelch domain (amino acids 315-598). The Kelch domain is the binding site with NRF2; CTR: C-terminal region (amino acids 599-624). The positions of the cysteine residues are indicated with yellow bars. The most commonly modified cysteine residues by sulfhydryl-reactive small molecules are shown in red.

electrophilic center(s) or have decreased electrophilicity has also supported the notion of the importance of sulfhydryl reactivity for induction of the KEAP1/NRF2 pathway and the heat shock response. In all cases, such alterations have led to either a complete loss of activity or a decrease in inducer potency. Thus, the sulfoxythiocarbamate group-containing analogues of sulforaphane which have much weaker sulfhydryl reactivity in comparison with the isothiocyanate group of sulforaphane are also less potent (5-10-fold) inducers of the NRF2-dependent enzyme NQO1 [93]. Among several series of naturally occurring and synthetic phenylpropanoids, the presence of a hydroxyl group at the ortho-position on the phenyl ring increases the chemical reactivity with sulfhydryl groups in parallel with enhancement of the inducer potency $[103,109,155]$. Compared to its hydroxylated analogue, bis(2-hydroxybenzylidene)acetone, the closely structurally related bis(benzylidene)acetone, reacts much more slowly with the sulfhydryl groups of glutathione and DTT and has much lower potency in inducing both the KEAP1/NRF2 pathway and the heat shock response [86, 109]. The recognition that inducer potency parallels sulfhydryl reactivity was a major driving force for the formation of the concept that the interaction with inducers and their intracellular sensor protein is based on chemical reactivity with cysteine residue(s) and not on receptor-ligand type of binding $[4,67,109,156]$.

Oleanolic acid which has the same pentacyclic skeleton, but lacks the electrophilic centers of the semisynthetic enoneor cyanoenone-bearing triterpenoids which induce NQO1 at nanomolar concentration, is completely inactive in this assay [134]. The cyclopentenone prostaglandins, which contain an $\alpha, \beta$-unsaturated carbonyl moiety in the cyclopentane ring, activate both HSF1 and NRF2, whereas other arachidonic acid metabolites that lack this electrophilic functional group do not have these effects $[74,157,158]$. In contrast to the large number of HSF1- and NRF2-target genes that are induced upon exposure to nitro-oleic acid, no induction is observed when cells are treated with its nonelectrophilic counterpart, oleic acid [75]. An analog of withaferin A, 2,3-dihydrowithaferin $\mathrm{A}$, which lacks the $\alpha, \beta$-unsaturated carbonyl moiety, is inactive in inducing HSF1 [94]. Similarly, the withanolide analogs, pubesenolide and viscosalactone
B which lack this sulfhydryl-reactive functional group, and curvularin which only differs from the active compound dehydrocurvularin by the absence of the $\alpha, \beta$-unsaturated carbonyl moiety, are also inactive [94]. Taken together, these findings imply that although not all small molecules that have the ability to react with sulfhydryl groups are inducers, reactivity with sulfhydryl groups constitutes the critical chemical signature responsible for induction of both cytoprotective pathways.

\section{Implications of Sulfhydryl Reactivity of Inducers of the KEAP1/NRF2 Pathway and the Heat Shock Response for the Identity of Their Protein Targets}

The common chemicals signature of reactivity with sulfhydryl groups among inducers of the KEAP1/NRF2 pathway and the heat shock response indicates that the protein targets for inducers of these pathways must possess reactive cysteine residues. The negative regulator of NRF2, KEAP1, is a multidomain homodimeric protein which has five distinct domains (Figure 2): (i) NTR: N-terminal region (amino acids 1-60); (ii) BTB: broad complex, Tramtrack, Bric-ábrac (amino acids 61-179) - the domain through which KEAP1 dimerizes; (iii) IVR: intervening region (amino acids $180-314)$ which is a particularly cysteine-rich region containing 8 cysteine residues among its 134 amino acids; (iv) Kelch domain (amino acids 315-598) - the domain through which KEAP1 binds to NRF2 and CUL3; and (v) CTR: Cterminal region (amino acids 599-624). There are 25 and 27 cysteine residues among the 624 amino acids of murine and human KEAP1, respectively. Nine of these cysteine residues (i.e., C23, C38, C151, C241, C273, C288, C297, C319, and C613) are flanked by basic amino acids and therefore predicted to have $p K_{a}$ values lower than that of free cysteine, favouring the formation of the thiolate anion at neutral $\mathrm{pH}$, and thus increasing the cysteine reactivity [159]. The NRF2 inducers sulforaphane and its sulfoxythiocarbamate derivative STCA have been shown to react with cysteine residues of KEAP1 using both purified recombinant protein as well as ectopically expressed KEAP1 isolated from cells 
that had been exposed to these compounds [18, 71, 93, $160,161]$. Mass spectroscopy and mutagenesis analyses have established that C151 in the BTB domain, and C273 and C288 in the IVR domain are critical for the repressor function of KEAP1; however, depending on the reaction conditions and the experimental system used, other cysteine residues have been also reported to be modified by sulforaphane, such as C38 in the N-terminal domain, C368, C489, and C583 in the Kelch domain, and C624 in the CTR $[160,161]$. KEAP1 reacts with tert-butyl quinone (tBQ), and the UVVis spectrum of the reaction product is identical to that of the product of the reaction of $\mathrm{tBQ}$ and DTT, implying that tBQ modifies cysteine residues of KEAP1 [20]. Mutation of C151 results in KEAP1 being a constitutive repressor of NRF2, which is unresponsive to activation by sulforaphane or tBHQ $[9,88]$. Substitution of C273 or C288 with either serine or alanine leads to a complete loss of repressor activity, and KEAP1 is unable to repress NRF2 even under basal conditions [74, 88, 162]. Transgenic mice expressing either C273A or C288A KEAP1 mutants have confirmed that these residues are required for the repressor function of KEAP1 under basal conditions [163].

The cysteine residues of KEAP1 form multiple discrete sensors for inducers. In a study conducted in zebrafish, a series of activators of NRF2 were classified into two groups: (i) inducers which react with C151 of KEAP1 (e.g., sulforaphane) and (ii) inducers which react with C273 (e.g., 15deoxy- $\Delta^{12,14}$-prostaglandin $\mathrm{J}_{2}$ ) [78]. Transgenic complementation rescue assays using embryonic fibroblasts and primary peritoneal macrophages isolated from mice expressing the C151S mutant of KEAP1 which were exposed to various inducers further confirmed these findings [76]. It was shown that $\mathrm{tBHQ}$, diethylmaleate, sulforaphane, and dimethylfumarate are sensed primarily by C151 of KEAP1, whereas the sensor(s) for 15-deoxy- $\Delta^{12,14}$-prostaglandin $\mathrm{J}_{2}, 2$-cyano-3,12 dioxooleana-1,9 diene-28-imidazolide (CDDO-Im), ebselen, nitro-oleic acid, and cadmium chloride are C151 independent. Recombinant KEAP1 reacts with monocyclic, tricyclic, and pentacyclic cyanoenones in vitro [19, 21, 134], and molecular docking studies using a model for the intervening region of KEAP1 and the $\mathrm{C} 1$ of the potential alkylation site of the triterpenoid CDDO-methylamide positioned against C226 of KEAP1 have suggested that C226 may be a target for this triterpenoid [164].

Using ectopically expressed KEAP1 in cultured mammalian cells exposed to a panel of inducers, McMahon and colleagues found that C151 and C288 of KEAP1 each form a distinct sensor with $\mathrm{C} 151$ responding to nitric oxide and C288 to alkenals, such as acrolein and 4-hydroxy-2-nonenal [71]. In addition, a third sensor was also identified which is formed by $\mathrm{H} 225, \mathrm{C} 226$, and $\mathrm{C} 613$ and was named the $\mathrm{Zn}^{++}$ sensor. A molecular model of the BTB domain of KEAP1 was constructed which revealed that C151 is in close spacial proximity with four basic amino acids, that is, K131, R135, and $\mathrm{K} 150$, and $\mathrm{H} 154$, an environment that could favor the thiolate formation at physiological $\mathrm{pH}$, thereby increasing the cysteine reactivity $[71,81]$. In strong agreement, when K131, $\mathrm{R} 135$, K150 were substituted with methionine residues, this mutant of KEAP1 had much lower sensitivity to sulforaphane and $\mathrm{tBHQ}$, and was almost completely unresponsive to nitric oxide [71]. In parallel, Fourquet et al. reported that exposure to hydrogen peroxide, to the nitric oxide donor spermine NONOate, to hypochlorous acid, or to $S$-nitrosocysteine causes formation of an intermolecular disulfide bridge linking two KEAP1 monomers via C151 [81]. It has also been suggested that one consequence of the binding of inducers to C151 may be the introduction of a steric clash with amino acid residues in the adjacent $\alpha$-helix which could alter the interaction between KEAP1 and CUL3 [165].

The finding that similar to the KEAP1/NRF2 pathway, sulfhydryl reactivity of small molecule inducers is important for activating the heat shock response is a much more recent discovery, and the identities of the sensor proteins are not firmly established. Depending on the biological system, cysteine-containing HSF1 or its negative regulators, Hsp90 or Hsp70, could potentially play this role. The HSF1 monomer has several distinct domains [61] (Figure 3): (i) DBD: Nterminal DNA-binding domain (amino acids 1-110); (ii) HRA/B: heptad repeat regions A and B (amino acids 130-203); (iii) $\mathrm{RD}$ : centrally located regulatory domain which negatively regulates the transactivating activity of HSF1 (amino acids 221-383); (iv) HR-C: heptad repeat region C (amino acids 384-409); and (v) TAD: C-terminal transactivation domain (amino acids 410-529). HSF1 trimerizes through intermolecular interactions between the HR-A/B regions, whereas trimerization is negatively regulated by intramolecular interactions between the HR-A/B heptad repeat region and the HR-C domains within the monomer. Mammalian HSF1 contains five conserved cysteine residues. It has been shown that activation of murine HSF1 by $\mathrm{H}_{2} \mathrm{O}_{2}$ is dependent on C35 and C105, which are located within the DNA-binding domain of the transcription factor and form a disulfide bridge in response to stress [82]. In the human HSF1, the corresponding pair of cysteines (i.e., C36 and C103) have been shown to form an intermolecular disulfide bond, a process which causes trimerization of the transcription factor and binding to heat shock elements in the promoter regions of heat shock genes [83]. In contrast, the formation of an intramolecular disulfide bond (in which C153, C373, and C378 participate) inhibits trimerization and DNA binding. These results are in agreement with earlier studies which showed that single mutation of $\mathrm{C} 153$ or double substitutions of C373 and C378 with serine residues prevented the formation of oxidized HSF1 in response to diamide [114], whereas treatment of HeLa cells with the glutathione-depleting agent L-buthionine sulfoximine promoted the formation of this oxidized species of HSF1 and blocked the heat-induced DNA binding activity of HSF1 [166].

In addition or alternative to directly modifying cysteine residues within HSF1, sulfhydryl-reactive activators may also react with cysteine residues of its negative regulators, Hsp90 and/or Hsp70. Inhibition of the chaperone activities of Hsp90 and/or Hsp70 may lead to the release of HSF1 and activation of the heat shock response. Indeed, induction of the heat shock response occurs commonly when Hsp90 activity is inhibited. Human Hsp90 is a homodimeric multidomain protein which contains four distinct regions: (i) N-terminal domain (amino acids 1-209) where ATP binds; (ii) a charged 


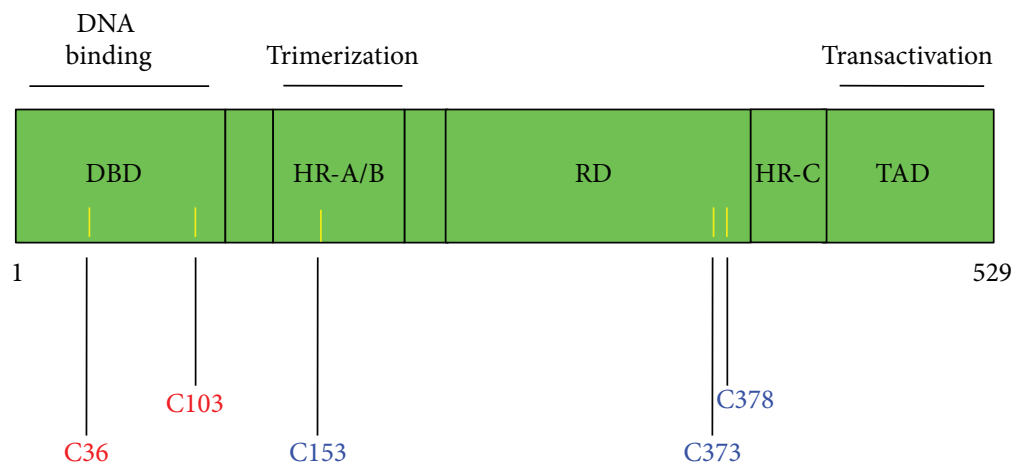

FIGURE 3: Domain structure of human HSF1. DBD: DNA-binding domain (amino acids 1-110); HR-A/B: heptad repeat regions A and B (amino acids 130-203); RD: regulatory domain (amino acids 221-383). This domain negatively regulates the transactivating activity of HSF1; HR-C: heptad repeat region C (amino acids 384-409); TAD: transactivation domain (amino acids 410-529). HSF1 trimerizes through intermolecular interactions between the HR-A/B regions. Trimerization is negatively regulated by intramolecular interactions between the HR-A/B heptad repeat region and the HR-C domains within the monomer. The positions of the cysteine residues are indicated with yellow bars. The cysteine residues which participate in activating intermolecular disulfide bonds are shown in red, and those which participate in inactivating intramolecular disulfide bonds are shown in blue.

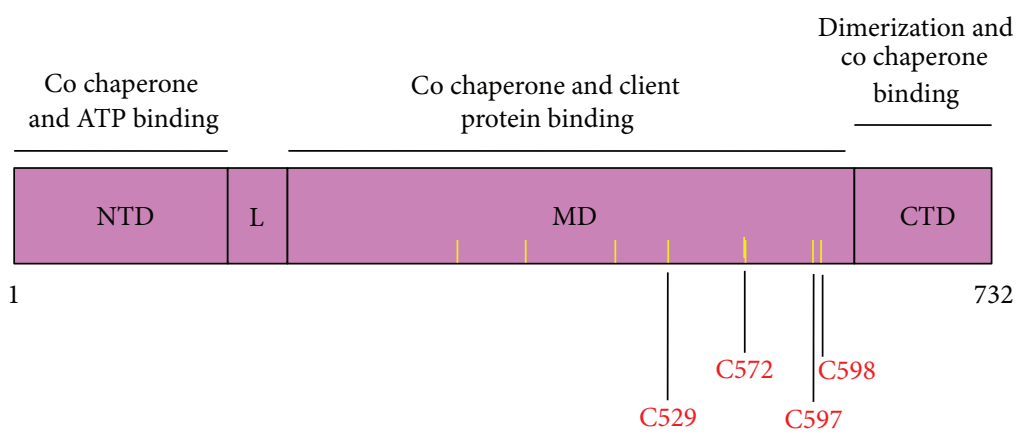

FIgURE 4: Domain structure of human Hsp90 $\alpha$. N-terminal domain (amino acids 1-209). This is the major site of ATP binding; L: flexible linker region (amino acids 210-271); MD: middle domain (amino acids 272-628) through which Hsp90 interacts with many of its client proteins; CTD: C-terminal domain (amino acids 629-732). Hsp90 forms a homodimer through the CTD. The positions of the cysteine residues are indicated with yellow bars. The most commonly modified cysteine residues by sulfhydryl-reactive small molecules are shown in red.

flexible linker region (amino acids 210-271); (iii) middle domain (amino acids 272-628) where Hsp90 interacts with many of its client proteins; and (iv) C-terminal domain (amino acids 629-732) through which the protein dimerizes (Figure 4) [167, 168]. The C-terminal domain contains a conserved MEEVD amino acid sequence implicated in binding to cochaperones with tetratricopeptide repeat (TRP) domains $[169,170]$. Posttranslational modifications of Hsp90 such as acetylation [171], phosphorylation [172, 173], and $S$-nitrosylation [174-177] have all been implicated in the regulation of the activity of the chaperone. Four of the seven cysteine residues of the mammalian Hsp90 are of particular importance for its chaperone function. It has been shown that in human Hsp90 $\alpha$, S-nitrosation at C597 inhibits the ATPase activity of the chaperone [177]. C521 and C589/590 in the rat Hsp90 $\beta$, corresponding to C529 and C597/C598 in Hsp90 $\alpha$, are highly reactive [178]. Substituting C597 in human Hsp90 $\alpha$ with $S$-nitrosation-mimicking residues, such as asparagine and aspartic acid, shifts the conformational equilibrium of the protein towards the open conformation, thus decreasing its chaperone activity [179]. This conclusion is also supported by molecular dynamics simulation approaches which have indicated that C597 is involved in regulating the conformation in Hsp90 [180]. Whereas C597 is conserved in eukaryotes and some bacteria, in yeast Hsp90, which is devoid of any cysteine residues, the corresponding amino acid is A577, and mutation to asparagine at this site strongly impairs the N-terminal association after ATP binding [179].

Recombinant human Hsp90 $\alpha$ has been shown to be modified by 4-hydroxy-2-nonenal at C572 [181], and C521 in recombinant $\mathrm{Hsp} 90 \beta$ forms a thiocarbamoylation conjugate with 6-methylsulfinylhexyl isothiocyanate [182]. Endogenous Hsp90 is also among the proteins that were found to be modified by 4 -hydroxy-2-nonenal $[72,183]$ and by its azido- and alkynyl-tagged derivatives as identified by the use of click chemistry and ex vivo biotinylation [184]. In addition, modifications in Hsp90 by 4-hydroxy-2-nonenal have been reported to occur in liver in a rat model of alcoholic liver disease [185]. By the use of proteomic and click chemistry 


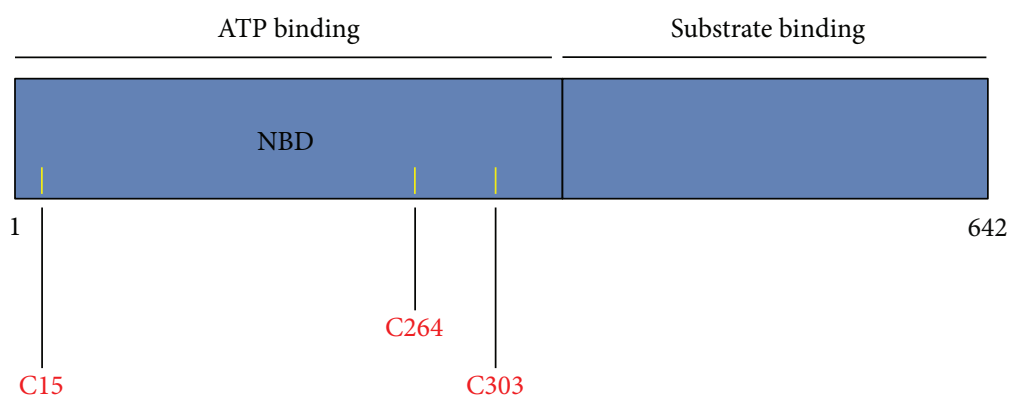

Figure 5: Domain structure of yeast Ssa1 Hsp70. The N-terminal half of the protein formS the nucleotide binding domain, and the C-terminal half is the substrate binding domain. The cysteine residues are indicated with yellow bars and their positions are shown in red.

approaches, Hsp90 was identified as being modified when HEK293 cells were exposed to the sulfoxythiocarbamate derivative of sulforaphane, STCA $[86,93]$. Sulforaphane was also recently shown to modify Hsp90 in vitro and to disrupt the interaction between Hsp90 and its co-chaperone Cdc37 and cause degradation of Hsp90 client proteins in pancreatic cancer cells [186]. Notably, in addition to reacting with cysteine, electrophilic compounds such as 4-hydroxy-2-nonenal can also react readily with histidine residues. Thus, the use of noncovalent affinity capture with a biotinyl-geldanamycin probe isolated both isoforms of the Hsp $90 \alpha$ and Hsp $90 \beta$ from human RKO colorectal cancer cells and enabled the detection of histidine adducts that were endogenously formed by the treatment with 4-hydroxy-2-nonenal [187]. Interestingly, no cysteine adducts on either Hsp90 $\alpha$ or Hsp90 $\beta$ were observed in this study.

HSF1 is also negatively regulated by Hsp70 and Hsp40. It is thus possible that structural alterations within these negative regulators may lead to the activation of HSF1 and induction of the heat shock response. Interestingly, modifications of both Hsp90 and Hsp70 are relatively commonly found after exposure to electrophiles. Hsp70 was found to be modified in cells which had been exposed to 4-hydroxy-2nonenal $[72,183]$ as well as to its azido- and alkynyl-tagged derivatives [184]. Hsp70 was also identified as one of the proteins that were modified by 4-hydroxy-2-nonenal in rat liver following chronic ethanol treatment [185]. Immunoprecipitation experiments have demonstrated that 4-hydroxy-2nonenal disrupts the interaction of ectopically expressed cMyc-tagged Hsp70 with HSF1 and induces the heat shock response [72]. Most recently, the Hsp70 chaperone Ssa1 was identified as the sensor for a range of sulfhydryl-reactive electrophiles which trigger the HSF1-mediated heat shock response in yeast (Saccharomyces cerevisiae) [73]. Notably, in yeast only Hsp70, but not Hsp90 or HSF1, contains cysteine residues: C15, C264, and C303, all of which are located within the nucleotide binding domain of Hsp70 Ssa1 (Figure 5). All three cysteines in Ssal can be modified by the alkylating agent $N$-ethylmaleimide, causing conformational changes and disruptions in interdomain communication, and ultimately resulting in inhibition of the ATP binding ability and protein folding activity of the chaperone [188-190]. Serine substitutions of C264 and C303, either individually or in combination, rendered this Ssal mutant unresponsive to celastrol, cadmium, and 4-hydroxy-2-nonenal, but interestingly, did not affect cellular responsiveness to thermal stress [73], indicating that the mechanisms for activation of HSF1 by sulfhydryl reactive agents and by thermal stress may not be the same. The same study showed that treatment of FLAG-tagged Ssa1-expressing cells with an alkyne derivative of 4-hydroxy-2-nonenal resulted in labelling of the wildtype Ssa1 by the electrophilic alkyne. In contrast, labelling was almost completely abolished in cells expressing the double C264S/C303S mutant and was decreased very substantially for the $\mathrm{C} 303 \mathrm{~S}$ mutant, suggesting that $\mathrm{C} 303$ is highly reactive and might be the prime site of electrophilic modification. Unlike the wildtype protein, a mutant in which C264 and C303 were substituted with aspartic acid, which mimics the sulfinic acid oxidation form of cysteine, failed to complement the HSF1 derepression phenotype of cells deficient for Ssa1. Taken together, these findings indicate that modification of C264 and C303 may result in structural alterations of Ssa1 ultimately leading to loss of its HSF1 repressor function. Mammalian Hsp70 was recently shown to be oxidized and inactivated by the redox-active compound, methylene blue at C306, and molecular modeling has suggested that oxidation of C306 also exposes C267 to modification, and it was proposed that both events contribute to loss of ATP binding and chaperone activity [191].

In addition to directly affecting the immediate repressing partners of HSF1, electrophilic HSF1 activators may also operate indirectly, by chemically modifying and thus altering the function of co-chaperones that form the Hsp90 complex machinery. Experimental evidence suggests that this could be the case for the co-chaperone $\mathrm{Cdc} 37$, which is involved in delivering client proteins to the Hsp90 complex. Cdc37 binds as a dimer to the $\mathrm{N}$-terminal domain of $\mathrm{Hsp} 90$ and arrests the ATPase cycle of the chaperone during client-protein loading $[192,193]$. The interaction between the ${ }^{1} \mathrm{H},{ }^{15} \mathrm{~N}$-labeled Nterminal domain of Hsp90 with unlabeled full-length Cdc37 has been examined in the absence of presence of celastrol using Heteronuclear Single Quantum Coherence (HSQC) NMR spectroscopy [194]. In combination with mutagenesis analysis and chemical modification (with $N$-ethylmaleimide) of the 9 cysteine residues of $\mathrm{Cdc} 37$, it was found that celastrol reacts with the 3 cysteine residues within the $\mathrm{N}$-terminal domain of $\mathrm{Cdc} 37$. This causes conformational changes in both the N-terminal and in the middle Hsp90-binding 
domains of Cdc37, and ultimately disrupts the Cdc37-Hsp90 complex.

\section{Cross-Talk between the KEAP1/NRF2 Pathway and the Heat Shock Response}

The KEAP1/NRF2 pathway and the heat shock response are two separate cytoprotective pathways. Induction of the NRF2-dependnet target enzyme NQO1 by sulfhydrylreactive agents of different structural types requires NRF2, but not HSF1, whereas induction of the HSF1-dependent protein $\mathrm{Hsp} 70$ by the same compounds depends on the presence of HSF1, but is independent of NRF2 [86]. Nonetheless, there is cross-talk between the two pathways. One example is rodent heme oxygenase 1, also known as Hsp32, which is induced both through activation of the KEAP1/NRF2 pathway and by heat shock [38, 195-199]. Interestingly, the presence of ARE sequences has been detected in the promoter regulatory regions of both murine and zebrafish $h s p 70$ [129]. Another recently reported intriguing link between the two pathways is the observation that Hsp90 and KEAP1 interact upon heat shock, leading to activation of NRF2 [200]. It is possible that by binding to KEAP1, Hsp90 protects the electrophilic stress sensor from potential damage during heat shock. It will be interesting to determine whether activation of NRF2, as a consequence of the interaction between KEAP1 and Hsp90 during heat shock, might be a contributing factor to the protective effect of heat shock against subsequent exposures to other forms of lethal cell stress.

\section{Concluding Remarks}

It should be noted that in vitro cysteine modifications of recombinant versions of the proteins that are targeted by sulfhydryl-reactive agents do not always correlate with the observed modifications in cellular protein targets isolated by the use of affinity probes. In addition, many compounds that react with sulfhydryl groups cause transient depletion of the intracellular glutathione and thioredoxin pools. It is therefore possible that it is the failure to maintain the physiological redox state of critical regulators, such as KEAP1, HSF1, Hap90, and/or Hsp70, rather than, or in addition to, their direct modifications by electrophilic small molecules, that activates the KEAP1/NRF2 pathway and the heat shock response. In the case of KEAP1, this possibility is highly unlikely as some inducers are active at concentrations in the low- and even subnanomolar range [19, 134], whereas glutathione is present in the cell at millimolar concentrations. Interestingly, the concentrations of sulfhydryl-reactive agents which induce the heat shock response are much higher than those that induce the KEAP1/NRF2 pathway. Thus, the possibility for such an indirect effect leading to the activation of HSF1 is more likely. However, it is also possible that the underlying reason for the different concentration requirements for the activation of the two pathways is the difference in the abundance of the protein targets. KEAP1 is a protein of an extremely low abundance. In sharp contrast, Hsp90 is highly abundant and constitutes $1-2 \%$ of the total cellular protein in a homeostatic unstressed cell. Another reason for the difference in the inducer concentration requirements could be a difference in the relative nucleophilicity of the cysteine residues of the target proteins and their accessibility for reaction with the electrophilic inducers. It could be proposed (Figure 1) that, at low concentrations, inducers are sensed first by the highly reactive cysteine residues of KEAP1, activating the KEAP1/NRF2 pathway. At higher concentrations, inducers affect the function of other targets, such as HSF1, Hsp90, Hsp70, or a co-chaperone within the Hsp90 complex machinery, leading to the activation of HSF1 and induction of the heat shock response. Future experiments are needed to test this proposal. However, it is clear at present that reactivity with sulfhydryl groups is a major determinant of the mechanism of action of small molecules dual activators of the KEAP1/NRF2 pathway and the heat shock response.

\section{Acknowledgments}

The author is very grateful to Rumen V. Kostov (Medical Research Institute, University of Dundee) for artwork and to Cancer Research UK (C20953/A10270), the Biotechnology and Biological Sciences Research Council (BBSRC, Project Grant BB/J007498/1), and Research Councils UK for the financial support.

\section{References}

[1] M. C. Gruhlke and A. J. Slusarenko, "The biology of reactive sulfur species (RSS)," Plant Physiology and Biochemistry, vol. 59, pp. 98-107, 2012.

[2] C. Jacob, E. Battaglia, T. Burkholz, D. Peng, D. Bagrel, and M. Montenarh, "Control of oxidative posttranslational cysteine modifications: from intricate chemistry to widespread biological and medical applications," Chemical Research in Toxicology, vol. 25, no. 3, pp. 588-604, 2012.

[3] P. Talalay, "Chemoprotection against cancer by induction of Phase 2 enzymes," BioFactors, vol. 12, no. 1-4, pp. 5-11, 2000.

[4] T. Prestera, Y. Zhang, S. R. Spencer, C. A. Wilczak, and P. Talalay, "The electrophile counterattack response: protection against neoplasia and toxicity," Advances in Enzyme Regulation, vol. 33, pp. 281-296, 1993.

[5] D. Malhotra, E. Portales-Casamar, A. Singh et al., "Global mapping of binding sites for Nrf2 identifies novel targets in cell survival response through chip-seq profiling and network analysis," Nucleic Acids Research, vol. 38, no. 17, pp. 5718-5734, 2010.

[6] K. Itoh, T. Chiba, S. Takahashi et al., "An Nrf2/small Maf heterodimer mediates the induction of phase II detoxifying enzyme genes through antioxidant response elements," Biochemical and Biophysical Research Communications, vol. 236, no. 2, pp. 313-322, 1997.

[7] M. McMahon, K. Itoh, M. Yamamoto, and J. D. Hayes, "Keap1dependent proteasomal degradation of transcription factor Nrf2 contributes to the negative regulation of antioxidant response element-driven gene expression," Journal of Biological Chemistry, vol. 278, no. 24, pp. 21592-21600, 2003.

[8] K. Itoh, N. Wakabayashi, Y. Katoh et al., "Keap1 represses nuclear activation of antioxidant responsive elements by $\mathrm{Nrf} 2$ 
through binding to the amino-terminal Neh2 domain," Genes and Development, vol. 13, no. 1, pp. 76-86, 1999.

[9] D. D. Zhang, S. C. Lo, J. V. Cross, D. J. Templeton, and M. Hannink, "Keap1 is a redox-regulated substrate adaptor protein for a Cul3-dependent ubiquitin ligase complex," Molecular and Cellular Biology, vol. 24, no. 24, pp. 10941-10953, 2004.

[10] M. Furukawa and Y. Xiong, "BTB protein keap1 targets antioxidant transcription factor Nrf2 for ubiquitination by the cullin 3-Roc1 ligase," Molecular and Cellular Biology, vol. 25, no. 1, pp. 162-171, 2005.

[11] A. Kobayashi, M. I. Kang, H. Okawa et al., "Oxidative stress sensor Keap1 functions as an adaptor for Cul3-based E3 ligase to regulate proteasomal degradation of Nrf2," Molecular and Cellular Biology, vol. 24, no. 16, pp. 7130-7139, 2004.

[12] P. Rada, A. I. Rojo, S. Chowdhry, M. McMahon, J. D. Hayes, and A. Cuadrado, "SCF/ $\beta$-TrCP promotes glycogen synthase kinase 3 -dependent degradation of the Nrf2 transcription factor in a Keap1-independent manner," Molecular and Cellular Biology, vol. 31, no. 6, pp. 1121-1133, 2011.

[13] P. Rada, A. I. Rojo, N. Evrard-Todeschi et al., "Structural and functional characterization of Nrf2 degradation by the glycogen synthase kinase 3/beta-TrCP axis," Molecular and Cellular Biology, vol. 32, no. 17, pp. 3486-3499, 2012.

[14] S. Chowdhry, Y. Zhang, M. McMahon, C. Sutherland, A. Cuadrado, and J. D. Hayes, "Nrf2 is controlled by two distinct beta-TrCP recognition motifs in its Neh6domain, one of which can be modulated by GSK-3 activity," Oncogene. In press.

[15] J. D. Hayes and M. McMahon, "NRF2 and KEAP1 mutations: permanent activation of an adaptive response in cancer," Trends in Biochemical Sciences, vol. 34, no. 4, pp. 176-188, 2009.

[16] L. Baird and A. T. Dinkova-Kostova, "The cytoprotective role of the Keap1-Nrf2 pathway," Archives of Toxicology, vol. 85, no. 4, pp. 241-272, 2011.

[17] N. F. Villeneuve, A. Lau, and D. D. Zhang, "Regulation of the Nrf2-keap1 antioxidant response by the ubiquitin proteasome system: an insight into cullin-ring ubiquitin ligases," Antioxidants and Redox Signaling, vol. 13, no. 11, pp. 1699-1712, 2010.

[18] A. T. Dinkova-Kostova, W. D. Holtzclaw, R. N. Cole et al., "Direct evidence that sulfhydryl groups of Keap1 are the sensors regulating induction of phase 2 enzymes that protect against carcinogens and oxidants," Proceedings of the National Academy of Sciences of the United States of America, vol. 99, no. 18, pp. 11908-11913, 2002.

[19] A. T. Dinkova-Kostova, P. Talalay, J. Sharkey et al., "An exceptionally potent inducer of cytoprotective enzymes: elucidation of the structural features that determine inducer potency and reactivity with Keap1," Journal of Biological Chemistry, vol. 285, no. 44, pp. 33747-33755, 2010.

[20] X. J. Wang, J. D. Hayes, L. G. Higgins, C. R. Wolf, and A. T. Dinkova-Kostova, "Activation of the NRF2 Signaling Pathway by Copper-Mediated Redox Cycling of Para- and Ortho-Hydroquinones," Chemistry and Biology, vol. 17, no. 1, pp. 75-85, 2010.

[21] S. Zheng, Y. R. S. Laxmi, E. David et al., "Synthesis, chemical reactivity as Michael acceptors, and biological potency of monocyclic cyanoenones, novel and highly potent antiinflammatory and cytoprotective agents," Journal of Medicinal Chemistry, vol. 55, no. 10, pp. 4837-4846, 2012.

[22] T. Nguyen, T. H. Rushmore, and C. B. Pickett, “Transcriptional regulation of a rat liver glutathione S-transferase Ya subunit gene. Analysis of the antioxidant response element and its activation by the phorbol ester 12-O-tetradecanoylphorbol-13acetate," Journal of Biological Chemistry, vol. 269, no. 18, pp. 13656-13662, 1994.

[23] L. V. Favreau and C. B. Pickett, "The rat quinone reductase antioxidant response element. Identification of the nucleotide sequence required for basal and inducible activity and detection of antioxidant response element-binding proteins in hepatoma and non-hepatoma cell lines," Journal of Biological Chemistry, vol. 270, no. 41, pp. 24468-24474, 1995.

[24] T. Nguyen, P. J. Sherratt, P. Nioi, C. S. Yang, and C. B. Pickett, "Nrf2 controls constitutive and inducible expression of ARE-driven genes through a dynamic pathway involving nucleocytoplasmic shuttling by Keap1," Journal of Biological Chemistry, vol. 280, no. 37, pp. 32485-32492, 2005.

[25] P. Nioi, M. McMahon, K. Itoh, M. Yamamoto, and J. D. Hayes, "Identification of a novel NRF2-regulated antioxidant response element (ARE) in the mouse $\mathrm{NAD}(\mathrm{P}) \mathrm{H}$ :quinone oxidoreductase 1 gene: reassessment of the ARE consensus sequence," Biochemical Journal, vol. 374, no. 2, pp. 337-348, 2003.

[26] R. S. Friling, A. Bensimon, Y. Tichauer, and V. Daniel, "Xen-obiotic-inducible expression of murine glutathione Stransferase Ya subunit gene is controlled by an electrophileresponsive element," Proceedings of the National Academy of Sciences of the United States of America, vol. 87, no. 16, pp. 6258-6262, 1990.

[27] T. H. Rushmore, R. G. King, K. E. Paulson, and C. B. Pickett, "Regulation of glutathione S-transferase Ya subunit gene expression: identification of a unique xenobiotic-responsive element controlling inducible expression by planar aromatic compounds," Proceedings of the National Academy of Sciences of the United States of America, vol. 87, no. 10, pp. 3826-3830, 1990.

[28] T. H. Rushmore and C. B. Pickett, "Transcriptional regulation of the rat glutathione S-transferase Ya subunit gene. Characterization of a xenobiotic-responsive element controlling inducible expression by phenolic antioxidants," Journal of Biological Chemistry, vol. 265, no. 24, pp. 14648-14653, 1990.

[29] J. D. Hayes, M. McMahon, S. Chowdhry, and A. T. DinkovaKostova, "Cancer chemoprevention mechanisms mediated through the keap1-Nrf2 pathway," Antioxidants and Redox Signaling, vol. 13, no. 11, pp. 1713-1748, 2010.

[30] A. M. Benson, R. P. Batzinger, S. Y. L. Ou, E. Bueding, Y. N. Cha, and P. Talalay, "Elevation of hepatic glutathione S-transferase activities and protection against mutagenic metabolites of benzo(a)pyrene by dietary antioxidants," Cancer Research, vol. 38, no. 12, pp. 4486-4495, 1978.

[31] A. M. Benson, Y. N. Cha, E. Bueding, H. S. Heine, and P. Talalay, "Elevation of extrahepatic glutathione S-transferase and epoxide hydratase activities by 2(3)-tert-butyl-4-hydroxyanisole," Cancer Research, vol. 39, no. 8, pp. 2971-2977, 1979.

[32] A. M. Benson, M. J. Hunkeler, and P. Talalay, "Increase of $\mathrm{NAD}(\mathrm{P}) \mathrm{H}$ :quinone reductase by dietary antioxidants: possible role in protection against carcinogenesis and toxicity," Proceedings of the National Academy of Sciences of the United States of America, vol. 77, no. 9, pp. 5216-5220, 1980.

[33] W. R. Pearson, J. J. Windle, and J. F. Morrow, "Increased synthesis of glutathione S-transferases in response to anticarcinogenic antioxidants. Cloning and measurement of messenger RNA," Journal of Biological Chemistry, vol. 258, no. 3, pp. 2052-2062, 1983. 
[34] P. Talalay and A. M. Benson, "Elevation of quinone reductase activity by anticarcinogenic antioxidants," Advances in Enzyme Regulation, vol. 20, pp. 287-300, 1982.

[35] R. T. Mulcahy, M. A. Wartman, H. H. Bailey, and J. J. Gipp, "Constitutive and $\beta$-naphthoflavone-induced expression of the human $\gamma$-glutamylcysteine synthetase heavy subunit gene is regulated by a distal antioxidant response element/TRE sequence," Journal of Biological Chemistry, vol. 272, no. 11, pp. 7445-7454, 1997.

[36] H. R. Moinova and R. Timothy Mulcahy, "An electrophile responsive element (EpRE) regulates $\beta$-naphthoflavone induction of the human $\gamma$-glutamylcysteine synthetase regulatory subunit gene: constitutive expression is mediated by an adjacent ap-1 site," Journal of Biological Chemistry, vol. 273, no. 24, pp. 14683-14689, 1998.

[37] H. R. Moinova and R. T. Mulcahy, "Up-regulation of the human $\gamma$-glutamylcysteine synthetase regulatory subunit gene involves binding of Nrf-2 to an electrophile responsive element," Biochemical and Biophysical Research Communications, vol. 261, no. 3, pp. 661-668, 1999.

[38] T. Prestera, P. Talalay, J. Alam, Y. I. Ahn, P. J. Lee, and A. M. Choi, "Parallel induction of heme oxygenase-1 and chemoprotective phase 2 enzymes by electrophiles and antioxidants: regulation by upstream antioxidant-responsive elements (ARE)," Molecular Medicine, vol. 1, no. 7, pp. 827-837, 1995.

[39] T. Primiano, T. W. Kensler, P. Kuppusamy, J. L. Zweier, and T. R. Sutter, "Induction of hepatic heme oxygenase-1 and ferritin in rats by cancer chemopreventive dithiolethiones," Carcinogenesis, vol. 17, no. 11, pp. 2291-2296, 1996.

[40] T. Primiano, J. A. Gastel, T. W. Kensler, and T. R. Sutter, "Isolation of cDNAs representing dithiolethione-responsive genes," Carcinogenesis, vol. 17, no. 11, pp. 2297-2303, 1996.

[41] T. Primiano, Y. Li, T. W. Kensler, M. A. Trush, and T. R. Sutter, "Identification of dithiolethione-inducible gene-1 as a leukotriene B4 12-hydroxydehydrogenase: implications for chemoprevention," Carcinogenesis, vol. 19, no. 6, pp. 999-1005, 1998.

[42] R. K. Thimmulappa, K. H. Mai, S. Srisuma, T. W. Kensler, M. Yamamoto, and S. Biswal, "Identification of Nrf2-regulated genes induced by the chemopreventive agent sulforaphane by oligonucleotide microarray," Cancer Research, vol. 62, no. 18, pp. 5196-5203, 2002.

[43] M. K. Kwak, N. Wakabayashi, K. Itoh, H. Motohashi, M. Yamamoto, and T. W. Kensler, "Modulation of gene expression by cancer chemopreventive dithiolethiones through the Keap1Nrf2 pathway: identification of novel gene clusters for cell survival," Journal of Biological Chemistry, vol. 278, no. 10, pp. 8135-8145, 2003.

[44] J. M. Lee, M. J. Calkins, K. Chan, Y. W. Kan, and J. A. Johnson, "Identification of the NF-E2-related factor-2-dependent genes conferring protection against oxidative stress in primary cortical astrocytes using oligonucleotide microarray analysis," Journal of Biological Chemistry, vol. 278, no. 14, pp. 12029-12038, 2003.

[45] Y. Mitsuishi, K. Taguchi, Y. Kawatani et al., "Nrf2 redirects glucose and glutamine into anabolic pathways in metabolic reprogramming," Cancer Cell, vol. 22, no. 1, pp. 66-79, 2012.

[46] K. C. Wu, J. Y. Cui, and C. D. Klaassen, "Beneficial role of nrf2 in regulating NADPH generation and consumption,” Toxicological Sciences, vol. 123, no. 2, pp. 590-600, 2011.

[47] K. Chan, R. Lu, J. C. Chang, and Y. W. Kan, "NRF2, a member of the NFE2 family of transcription factors, is not essential for murine erythropoiesis, growth, and development," Proceedings of the National Academy of Sciences of the United States of America, vol. 93, no. 24, pp. 13943-13948, 1996.

[48] T. W. Kensler, N. Wakabayashi, and S. Biswal, "Cell survival responses to environmental stresses via the Keap1-Nrf2-ARE pathway," Annual Review of Pharmacology and Toxicology, vol. 47, pp. 89-116, 2007.

[49] T. W. Kensler and N. Wakabayashi, "Nrf2: friend or foe for chemoprevention?” Carcinogenesis, vol. 31, no. 1, pp. 90-99, 2009.

[50] M. Ramos-Gomez, M. K. Kwak, P. M. Dolan et al., "Sensitivity to carcinogenesis is increased and chemoprotective efficacy of enzyme inducers is lost in nrf2 transcription factor-deficient mice," Proceedings of the National Academy of Sciences of the United States of America, vol. 98, no. 6, pp. 3410-3415, 2001.

[51] S. Chowdhry, M. H. Nazmy, P. J. Meakin et al., "Loss of Nrf2 markedly exacerbates nonalcoholic steatohepatitis," Free Radical Biology and Medicine, vol. 48, no. 2, pp. 357-371, 2010.

[52] T. Ohta, K. Iijima, M. Miyamoto et al., "Loss of Keap1 function activates Nrf2 and provides advantages for lung cancer cell growth,” Cancer Research, vol. 68, no. 5, pp. 1303-1309, 2008.

[53] T. Shibata, T. Ohta, K. I. Tong et al., "Cancer related mutations in NRF2 impair its recognition by Keap1-Cul3 E3 ligase and promote malignancy," Proceedings of the National Academy of Sciences of the United States of America, vol. 105, no. 36, pp. 13568-13573, 2008.

[54] P. A. Konstantinopoulos, D. Spentzos, E. Fountzilas et al., "Keap1 mutations and Nrf2 pathway activation in epithelial ovarian cancer," Cancer Research, vol. 71, no. 15, pp. 5081-5089, 2011.

[55] T. Shibata, A. Kokubu, M. Gotoh et al., "Genetic Alteration of Keap1 Confers Constitutive Nrf2 Activation and Resistance to Chemotherapy in Gallbladder Cancer," Gastroenterology, vol. 135, no. 4, pp. 1358-1368, 2008.

[56] A. Singh, V. Misra, R. K. Thimmulappa et al., "Dysfunctional KEAP1-NRF2 interaction in non-small-cell lung cancer," PLoS Medicine, vol. 3, no. 10, article e420, 2006.

[57] Y. R. Kim, J. E. Oh, M. S. Kim et al., "Oncogenic NRF2 mutations in squamous cell carcinomas of oesophagus and skin," Journal of Pathology, vol. 220, no. 4, pp. 446-451, 2010.

[58] P. S. Hammerman, M. S. Lawrence, D. Voet et al., "Comprehensive genomic characterization of squamous cell lung cancers," Nature, vol. 489, no. 7417, pp. 519-525, 2012.

[59] G. M. Denicola, F. A. Karreth, T. J. Humpton et al., "Oncogeneinduced Nrf2 transcription promotes ROS detoxification and tumorigenesis," Nature, vol. 475, no. 7354, pp. 106-110, 2011.

[60] M. Åkerfelt, R. I. Morimoto, and L. Sistonen, "Heat shock factors: integrators of cell stress, development and lifespan," Nature Reviews Molecular Cell Biology, vol. 11, no. 8, pp. 545-555, 2010.

[61] J. Anckar and L. Sistonen, "Regulation of HSF1 function in the heat stress response: implications in aging and disease," Annual Review of Biochemistry, vol. 80, pp. 1089-1115, 2011.

[62] A. Ali, S. Bharadwaj, R. O'Carroll, and N. Ovsenek, "HSP90 interacts with and regulates the activity of heat shock factor 1 in Xenopus oocytes," Molecular and Cellular Biology, vol. 18, no. 9, pp. 4949-4960, 1998.

[63] J. Zou, Y. Guo, T. Guettouche, D. F. Smith, and R. Voellmy, "Repression of heat shock transcription factor HSF1 activation by HSP90 (HSP90 complex) that forms a stress-sensitive complex with HSF1," Cell, vol. 94, no. 4, pp. 471-480, 1998. 
[64] K. Richter, M. Haslbeck, and J. Buchner, "The heat shock response: life on the verge of death," Molecular Cell, vol. 40, no. 2, pp. 253-266, 2010.

[65] M. L. Mendillo, S. Santagata, M. Koeva et al., "HSF1 drives a transcriptional program distinct from heat shock to support highly malignant human cancers," Cell, vol. 150, no. 3, pp. 549-562, 2012.

[66] S. Santagata, R. Hu, N. U. Lin et al., "High levels of nuclear heat-shock factor 1 (HSF1) are associated with poor prognosis in breast cancer," Proceedings of the National Academy of Sciences of the United States of America, vol. 108, no. 45, pp. 18378-18383, 2011.

[67] P. Talalay, M. J. De Long, and H. J. Prochaska, "Identification of a common chemical signal regulating the induction of enzymes that protect against chemical carcinogenesis," Proceedings of the National Academy of Sciences of the United States of America, vol. 85, no. 21, pp. 8261-8265, 1988.

[68] H. J. Prochaska and A. B. Santamaria, "Direct measurement of $\mathrm{NAD}(\mathrm{P}) \mathrm{H}$ : quinone reductase from cells cultured in microtiter wells: a screening assay for anticarcinogenic enzyme inducers," Analytical Biochemistry, vol. 169, no. 2, pp. 328-336, 1988.

[69] J. W. Fahey, A. T. Dinkova-Kostova, K. K. Stephenson, and P. Talalay, "The "Prochaska" Microtiter Plate Bioassay for Inducers of NQO1," Methods in Enzymology, vol. 382, pp. 243-258, 2004.

[70] A. T. Dinkova-Kostova, J. W. Fahey, and P. Talalay, "Chemical Structures of Inducers of Nicotinamide Quinone Oxidoreductase 1 (NQO1)," Methods in Enzymology, vol. 382, pp. 423-448, 2004.

[71] M. McMahon, D. J. Lamont, K. A. Beattie, and J. D. Hayes, "Keap1 perceives stress via three sensors for the endogenous signaling molecules nitric oxide, zinc, and alkenals," Proceedings of the National Academy of Sciences of the United States of America, vol. 107, no. 44, pp. 18838-18843, 2010.

[72] A. T. Jacobs and L. J. Marnett, "Heat shock factor 1 attenuates 4hydroxynonenal-mediated apoptosis: critical role for heat shock protein 70 induction and stabilization of Bcl-XL," Journal of Biological Chemistry, vol. 282, no. 46, pp. 33412-33420, 2007.

[73] Y. Wang, P. A. Gibney, J. D. West, and K. A. Morano, “The yeast Hsp70 Ssa1 is a sensor for activation of the heat shock response by thiol-reactive compounds," Molecular Biology of the Cell, vol. 23, no. 17, pp. 3290-3298, 2012.

[74] A. L. Levonen, A. Landar, A. Ramachandran et al., "Cellular mechanisms of redox cell signalling: role of cysteine modification in controlling antioxidant defences in response to electrophilic lipid oxidation products," Biochemical Journal, vol. 378, no. 2, pp. 373-382, 2004.

[75] E. Kansanen, H. K. Jyrkkänen, O. L. Volger et al., "Nrf2dependent and -independent responses to nitro-fatty acids in human endothelial cells: identification of heat shock response as the major pathway activated by nitro-oleic acid," Journal of Biological Chemistry, vol. 284, no. 48, pp. 33233-33241, 2009.

[76] K. Takaya, T. Suzuki, H. Motohashi et al., "Validation of the multiple sensor mechanism of the Keap1-Nrf2 system," Free Radical Biology \& Medicine, vol. 53, no. 4, pp. 817-827, 2012.

[77] B. Zingarelli, P. W. Hake, P. Mangeshkar et al., "Diverse cardioprotective signaling mechanisms of peroxisome proliferatoractivated receptor- $\gamma$ ligands, 15 -deoxy- $\Delta 12,14$ - prostaglandin $\mathrm{J} 2$ and ciglitazone, in reperfusion injury: role of nuclear factor$\kappa \mathrm{B}$, heat shock factor 1, and Akt," Shock, vol. 28, no. 5, pp. 554-563, 2007.

[78] M. Kobayashi, L. Li, N. Iwamoto et al., "The antioxidant defense system Keap1-Nrf2 comprises a multiple sensing mechanism for responding to a wide range of chemical compounds," Molecular and Cellular Biology, vol. 29, no. 2, pp. 493-502, 2009.

[79] E. Kansanen, A. M. Kivelä, and A. L. Levonen, "Regulation of Nrf2-dependent gene expression by 15 -deoxy- $\Delta 12,14$ prostaglandin J2," Free Radical Biology and Medicine, vol. 47, no. 9, pp. 1310-1317, 2009.

[80] C. A. Thompson and P. C. Burcham, "Genome-wide transcriptional responses to acrolein," Chemical Research in Toxicology, vol. 21, no. 12, pp. 2245-2256, 2008.

[81] S. Fourquet, R. Guerois, D. Biard, and M. B. Toledano, "Activation of NRF2 by nitrosative agents and $\mathrm{H} 2 \mathrm{O} 2$ involves KEAP1 disulfide formation," Journal of Biological Chemistry, vol. 285, no. 11, pp. 8463-8471, 2010.

[82] S. G. Ahn and D. J. Thiele, "Redox regulation of mammalian heat shock factor 1 is essential for Hsp gene activation and protection from stress," Genes and Development, vol. 17, no. 4, pp. 516-528, 2003.

[83] M. Lu, H. E. Kim, C. R. Li et al., "Two distinct disulfide bonds formed in human heat shock transcription factor 1 act in opposition to regulate its DNA binding activity," Biochemistry, vol. 47 , no. 22 , pp. $6007-6015,2008$.

[84] A. Trott, J. D. West, L. Klaić et al., "Activation of heat shock and antioxidant responses by the natural product celastrol: transcriptional signatures of a thiol-targeted molecule," Molecular Biology of the Cell, vol. 19, no. 3, pp. 1104-1112, 2008.

[85] S. D. Westerheide, J. D. Bosman, B. N. A. Mbadugha et al., "Celastrols as inducers of the heat shock response and cytoprotection," Journal of Biological Chemistry, vol. 279, no. 53, pp. 56053-56060, 2004.

[86] Y. Zhang, Y. H. Ahn, I. J. Benjamin et al., "HSF1-dependent upregulation of Hsp70 by sulfhydryl-reactive inducers of the KEAP1/NRF2/ARE pathway," Chemistry \& Biology, vol. 18, no. 11, pp. 1355-1361, 2011.

[87] W. Y. Seo, A. R. Goh, S. M. Ju et al., "Celastrol induces expression of heme oxygenase-1 through ROS/Nrf2/ARE signaling in the HaCaT cells," Biochemical and Biophysical Research Communications, vol. 407, no. 3, pp. 535-540, 2011.

[88] D. D. Zhang and M. Hannink, "Distinct Cysteine Residues in Keap1 Are Required for Keap1-Dependent Ubiquitination of $\mathrm{Nrf} 2$ and for Stabilization of Nrf2 by Chemopreventive Agents and Oxidative Stress," Molecular and Cellular Biology, vol. 23, no. 22, pp. 8137-8151, 2003.

[89] N. Gan, Y. C. Wu, M. Brunet et al., "Sulforaphane activates heat shock response and enhances proteasome activity through upregulation of Hsp27," Journal of Biological Chemistry, vol. 285, no. 46, pp. 35528-35536, 2010.

[90] R. Hu, C. Xu, G. Shen et al., "Gene expression profiles induced by cancer chemopreventive isothiocyanate sulforaphane in the liver of C57BL/6J mice and C57BL/6J/Nrf2 (-/-) mice," Cancer Letters, vol. 243, no. 2, pp. 170-192, 2006.

[91] Y. Zhang, P. Talalay, C. G. Cho, and G. H. Posner, "A major inducer of anticarcinogenic protective enzymes from broccoli: isolation and elucidation of structure," Proceedings of the National Academy of Sciences of the United States of America, vol. 89, no. 6, pp. 2399-2403, 1992.

[92] R. Hu, C. Xu, G. Shen et al., "Identification of Nrf2-regulated genes induced by chemopreventive isothiocyanate PEITC by oligonucleotide microarray," Life Sciences, vol. 79, no. 20, pp. 1944-1955, 2006.

[93] Y. H. Ahn, Y. Hwang, H. Liu et al., "Electrophilic tuning of the chemoprotective natural product sulforaphane," Proceedings of 
the National Academy of Sciences of the United States of America, vol. 107, no. 21, pp. 9590-9595, 2010.

[94] S. Santagata, Y. M. Xu, E. M. Wijeratne et al., "Using the heatshock response to discover anticancer compounds that target protein homeostasis," ACS Chemical Biology, vol. 7, no. 2, pp. 340-349, 2012.

[95] Y. Yu, A. Hamza, T. Zhang et al., "Withaferin A targets heat shock protein 90 in pancreatic cancer cells," Biochemical Pharmacology, vol. 79, no. 4, pp. 542-551, 2010.

[96] A. Sood, R. Mathew, and H. Trachtman, "Cytoprotective effect of curcumin in human proximal tubule epithelial cells exposed to Shiga toxin," Biochemical and Biophysical Research Communications, vol. 283, no. 1, pp. 36-41, 2001.

[97] M. Kanitkar and R. R. Bhonde, "Curcumin treatment enhances islet recovery by induction of heat shock response proteins, Hsp70 and heme oxygenase-1, during cryopreservation," Life Sciences, vol. 82, no. 3-4, pp. 182-189, 2008.

[98] S. Khan and J. J. Heikkila, "Curcumin-induced inhibition of proteasomal activity, enhanced HSP accumulation and the acquisition of thermotolerance in Xenopus laevis A6 cells," Comparative Biochemistry and Physiology, vol. 158, no. 4, pp. 566-576, 2011.

[99] S. Q. Shen, Y. Zhang, J. J. Xiang, and C. L. Xiong, "Protective effect of curcumin against liver warm ischemia/reperfusion injury in rat model is associated with regulation of heat shock protein and antioxidant enzymes," World Journal of Gastroenterology, vol. 13, no. 13, pp. 1953-1961, 2007.

[100] K. Kato and H. Ito, "Stimulation of the stress-induced expression of stress proteins by curcumin in cultured cells and in rat tissues in vivo," Cell Stress \& Chaperones, vol. 3, no. 3, pp. 152-160, 1998.

[101] L. X. Wu, J. H. Xu, X. W. Huang, K. Z. Zhang, C. X. Wen, and Y. Z. Chen, "Down-regulation of $\mathrm{p} 210 \mathrm{bcr} / \mathrm{abl}$ by curcumin involves disrupting molecular chaperone functions of Hsp90," Acta Pharmacologica Sinica, vol. 27, no. 6, pp. 694-699, 2006.

[102] H. W. Chen, S. L. Yu, J. J. W. Chen et al., "Anti-Invasive Gene Expression Profile of Curcumin in Lung Adenocarcinoma Based on a High Throughput Microarray Analysis," Molecular Pharmacology, vol. 65, no. 1, pp. 99-110, 2004.

[103] A. T. Dinkova-Kostova and P. Talalay, "Relation of structure of curcumin analogs to their potencies as inducers of Phase 2 detoxification enzymes," Carcinogenesis, vol. 20, no. 5, pp. 911-914, 1999.

[104] L. Charoensuk, P. Pinlaor, S. Prakobwong et al., "Curcumin induces a nuclear factor-erythroid 2-related factor 2-driven response against oxidative and nitrative stress after praziquantel treatment in liver fluke-infected hamsters," International Journal for Parasitology, vol. 41, no. 6, pp. 615-626, 2011.

[105] R. Manikandan, M. Beulaja, R. Thiagarajan, and M. Arumugam, "Effect of curcumin on the modulation of $\alpha \mathrm{A}$ - and $\alpha \mathrm{B}$-crystallin and heat shock protein 70 in selenium-induced cataractogenesis in Wistar rat pups," Molecular Vision, vol. 17, pp. 388-394, 2011.

[106] C. Yang, X. Zhang, H. Fan, and Y. Liu, "Curcumin upregulates transcription factor Nrf2, HO-1 expression and protects rat brains against focal ischemia," Brain Research, vol. 1282, pp. 133-141, 2009.

[107] G. Shen, C. Xu, R. Hu et al., "Modulation of nuclear factor E2-related factor 2-mediated gene expression in mice liver and small intestine by cancer chemopreventive agent curcumin," Molecular Cancer Therapeutics, vol. 5, no. 1, pp. 39-51, 2006.

[108] S. Khan and J. J. Heikkila, "Curcumin-induced inhibition of proteasomal activity, enhanced HSP accumulation and the acquisition of thermotolerance in Xenopus laevis A6 cells," Comparative Biochemistry and Physiology A, vol. 158, no. 4, pp. 566-576, 2011.

[109] A. T. Dinkova-Kostova, M. A. Massiah, R. E. Bozak, R. J. Hicks, and P. Talalay, "Potency of Michael reaction acceptors as inducers of enzymes that protect against carcinogenesis depends on their reactivity with sulfhydryl groups," Proceedings of the National Academy of Sciences of the United States of America, vol. 98, no. 6, pp. 3404-3409, 2001.

[110] N. A. Smirnova, R. E. Haskew-Layton, M. Basso et al., "Development of Neh2-luciferase reporter and its application for high throughput screening and real-time monitoring of $\mathrm{Nrf} 2$ activators," Chemistry and Biology, vol. 18, no. 6, pp. 752-765, 2011.

[111] B. Zhang, Q. Au, I. S. Yoon et al., "Identification of smallmolecule HSF1 amplifiers by high content screening in protection of cells from stress induced injury," Biochemical and Biophysical Research Communications, vol. 390, no. 3, pp. 925-930, 2009.

[112] R. L. Matts, G. E. L. Brandt, Y. Lu et al., "A systematic protocol for the characterization of Hsp90 modulators," Bioorganic and Medicinal Chemistry, vol. 19, no. 1, pp. 684-692, 2011.

[113] H. Hieronymus, J. Lamb, K. N. Ross et al., "Gene expression signature-based chemical genomic prediction identifies a novel class of HSP90 pathway modulators," Cancer Cell, vol. 10, no. 4, pp. 321-330, 2006.

[114] D. J. Manalo, Z. Lin, and A. Y. C. Liu, "Redox-dependent regulation of the conformation and function of human heat shock factor 1," Biochemistry, vol. 41, no. 8, pp. 2580-2588, 2002.

[115] K. L. Hamilton, S. Gupta, and A. A. Knowlton, "Estrogen and regulation of heat shock protein expression in female cardiomyocytes: cross-talk with $\mathrm{NF} \kappa \mathrm{B}$ signaling," Journal of Molecular and Cellular Cardiology, vol. 36, no. 4, pp. 577-584, 2004.

[116] K. L. Hamilton, F. N. Mbai, S. Gupta, and A. A. Knowlton, "Estrogen, heat shock proteins, and $\mathrm{NF} \kappa \mathrm{B}$ in human vascular endothelium," Arteriosclerosis, Thrombosis, and Vascular Biology, vol. 24, no. 9, pp. 1628-1633, 2004.

[117] T. Satoh, T. Rezaie, M. Seki et al., "Dual neuroprotective pathways of a pro-electrophilic compound via HSF-1-activated heat-shock proteins and Nrf2-activated phase 2 antioxidant response enzymes," Journal of Neurochemistry, vol. 119, no. 3, pp. 569-578, 2011.

[118] J. N. Saykally, L. Rachmany, H. Hatic et al., "The nuclear factor erythroid 2-like 2 activator, tert-butylhydroquinone, improves cognitive performance in mice after mild traumatic brain injury," Neuroscience, vol. 223, pp. 305-314, 2012.

[119] H. Hatic, M. J. Kane, J. N. Saykally, and B. A. Citron, "Modulation of transcription factor Nrf2 in an in vitro model of traumatic brain injury," Journal of Neurotrauma, vol. 29, no. 6, pp. 1188-1196, 2012.

[120] S. Khalil, J. Luciano, W. Chen, and A. Y. C. Liu, "Dynamic regulation and involvement of the heat shock transcriptional response in arsenic carcinogenesis," Journal of Cellular Physiology, vol. 207, no. 2, pp. 562-569, 2006.

[121] L. M. Del Razo, B. Quintanilla-Vega, E. Brambila-Colombres, E. S. Calderón-Aranda, M. Manno, and A. Albores, "Stress proteins induced by arsenic," Toxicology and Applied Pharmacology, vol. 177, no. 2, pp. 132-148, 2001.

[122] J. A. Barnes, B. W. Collins, D. J. Dix, and J. W. Allen, "Effects of heat shock protein 70 (Hsp70) on arsenite-induced 
genotoxicity," Environmental and Molecular Mutagenesis, vol. 40, no. 4, pp. 236-242, 2002.

[123] B. Fauconneau, V. Petegnief, C. Sanfeliu, A. Piriou, and A. M. Planas, "Induction of heat shock proteins (HSPs) by sodium arsenite in cultured astrocytes and reduction of hydrogen peroxide-induced cell death," Journal of Neurochemistry, vol. 83, no. 6, pp. 1338-1348, 2002.

[124] J. Liu, M. B. Kadiiska, Y. Liu, T. Lu, W. Qu, and M. P. Waalkes, "Stress-related gene expression in mice treated with inorganic arsenicals," Toxicological Sciences, vol. 61, no. 2, pp. 314-320, 2001.

[125] M. Bengleil, S. Hassaneen, and J. R. Fry, "Induction of anoikis by sodium arsenite in rat hepatoma FGC4 cells: implications for assessment of regulation of heat shock protein 70," Toxicology Mechanisms and Methods, vol. 22, no. 8, pp. 611-616, 2012.

[126] L. Bhagat, V. P. Singh, R. K. Dawra, and A. K. Saluja, "Sodium arsenite induces heat shock protein 70 expression and protects against secretagogue-induced trypsinogen and NF- $\kappa \mathrm{B}$ activation," Journal of Cellular Physiology, vol. 215, no. 1, pp. 37-46, 2008.

[127] Z. Rakonczay, Y. Mándi, J. Kaszaki et al., "Induction of HSP72 by sodium arsenite fails to protect against cholecystokininoctapeptide-induced acute pancreatitis in rats," Digestive Diseases and Sciences, vol. 47, no. 7, pp. 1594-1603, 2002.

[128] M. K. Kwak, M. Ramos-Gomez, N. Wakabayashi, and T. W. Kensler, "Chemoprevention by 1,2-Dithiole-3-Thiones Through Induction of NQO1 and Other Phase 2 Enzymes," Methods in Enzymology, vol. 382, pp. 414-423, 2004.

[129] D. V. Almeida, B. F. da Silva Nornberg, L. A. Geracitano, D. M. Barros, J. M. Monserrat, and L. F. Marins, "Induction of phase II enzymes and hsp70 genes by copper sulfate through the electrophile-responsive element (EpRE): insights obtained from a transgenic zebrafish model carrying an orthologous EpRE sequence of mammalian origin," Fish Physiology and Biochemistry, vol. 36, no. 3, pp. 347-353, 2010.

[130] B. J. Wu, R. E. Kingston, and R. I. Morimoto, "Human HSP70 promoter contains at least two distinct regulatory domains," Proceedings of the National Academy of Sciences of the United States of America, vol. 83, no. 3, pp. 629-633, 1986.

[131] K. Mitani, H. Fujita, S. Sassa, and A. Kappas, "Activation of heme oxygenase and heat shock protein 70 genes by stress in human hepatoma cells," Biochemical and Biophysical Research Communications, vol. 166, no. 3, pp. 1429-1434, 1990.

[132] B. Calamini, M. C. Silva, F. Madoux et al., "Small-molecule proteostasis regulators for protein conformational diseases," Nature Chemical Biology, vol. 8, no. 2, pp. 185-196, 2012.

[133] T. Guettouche, F. Boellmann, W. S. Lane, and R. Voellmy, "Analysis of phosphorylation of human heat shock factor 1 in cells experiencing a stress," BMC Biochemistry, vol. 6, article 4, 2005.

[134] A. T. Dinkova-Kostova, K. T. Liby, K. K. Stephenson et al., "Extremely potent triterpenoid inducers of the phase 2 response: correlations of protection against oxidant and inflammatory stress," Proceedings of the National Academy of Sciences of the United States of America, vol. 102, no. 12, pp. 4584-4589, 2005.

[135] M. B. Sporn, K. T. Liby, M. M. Yore, L. Fu, J. M. Lopchuk, and G. W. Gribble, "New synthetic triterpenoids: potent agents for prevention and treatment of tissue injury caused by inflammatory and oxidative stress," Journal of Natural Products, vol. 74, no. 3, pp. 537-545, 2011.
[136] R. V. Bensasson, V. Zoete, G. Berthier, P. Talalay, and A. T. Dinkova-Kostova, "Potency ranking of triterpenoids as inducers of a cytoprotective enzyme and as inhibitors of a cellular inflammatory response via their electron affinity and their electrophilicity index," Chemico-Biological Interactions, vol. 186, no. 2, pp. 118-126, 2010.

[137] S. Kalra, E. V. Knatko, Y. Zhang, T. Honda, M. Yamamoto, and A. T. Dinkova-Kostova, "Highly potent activation of Nrf2 by topical tricyclic bis(cyano enone): implications for protection against UV radiation during thiopurine therapy," Cancer Prevention Research, vol. 5, no. 7, pp. 973-981, 2012.

[138] K. Liby, M. M. Yore, B. D. Roebuck et al., "A novel acetylenic tricyclic bis-(cyano enone) potently induces phase 2 cytoprotective pathways and blocks liver carcinogenesis induced by aflatoxin," Cancer Research, vol. 68, no. 16, pp. 6727-6733, 2008.

[139] H. J. Prochaska, M. J. De Long, and P. Talalay, "On the mechanisms of induction of cancer-protective enzymes: a unifying proposal," Proceedings of the National Academy of Sciences of the United States of America, vol. 82, no. 23, pp. 8232-8236, 1985.

[140] R. V. Bensasson, V. Zoete, A. T. Dinkova-Kostova, and P. Talalay, "Two-step mechanism of induction of the gene expression of a prototypic cancer-protective enzyme by diphenols," Chemical Research in Toxicology, vol. 21, no. 4, pp. 805-812, 2008.

[141] D. Sumi, Y. Numasawa, A. Endo, N. Iwamoto, and Y. Kumagai, "Catechol estrogens mediated activation of Nrf2 through covalent modification of its quinone metabolite to Keap1," Journal of Toxicological Sciences, vol. 34, no. 6, pp. 627-635, 2009.

[142] L. A. Stocken and R. H. Thompson, "British anti-lewisite: 2. Dithiol compounds as antidotes for arsenic," The Biochemical Journal, vol. 40, no. 4, pp. 535-548, 1946.

[143] W. Hur, Z. Sun, T. Jiang et al., "A small-molecule inducer of the antioxidant response element," Chemistry and Biology, vol. 17, no. 5, pp. 537-547, 2010.

[144] H. Liu, R. Lightfoot, and J. L. Stevens, "Activation of heat shock factor by alkylating agents is triggered by glutathione depletion and oxidation of protein thiols," Journal of Biological Chemistry, vol. 271, no. 9, pp. 4805-4812, 1996.

[145] E. L. Schaefer, R. I. Morimoto, N. G. Theodorakis, and J. Seidenfeld, "Chemical specificity for induction of stress response genes by DNA-damaging drugs in human adenocarcinoma cells," Carcinogenesis, vol. 9, no. 10, pp. 1733-1738, 1988.

[146] R. A. Kroes, K. Abravaya, J. Seidenfeld, and R. I. Morimoto, "Selective activation of human heat shock gene transcription by nitrosourea antitumor drugs mediated by isocyanate-induced damage and activation of heat shock transcription factor," Proceedings of the National Academy of Sciences of the United States of America, vol. 88, no. 11, pp. 4825-4829, 1991.

[147] Q. Chen, K. Yu, and J. L. Stevens, "Regulation of the cellular stress response by reactive electrophiles. The role of covalent binding and cellular thiols in transcriptional activation of the 70-kilodalton heat shock protein gene by nephrotoxic cysteine conjugates," Journal of Biological Chemistry, vol. 267, no. 34, pp. 24322-24327, 1992.

[148] Y. J. Moon, D. A. Brazeau, and M. E. Morris, "Dietary phenethyl isothiocyanate alters gene expression in human breast cancer cells," Evidence-based Complementary and Alternative Medicine, vol. 2011, Article ID 462525, 2011.

[149] S. H. Kim, S. I. Han, S. Y. Oh, H. Y. Chung, H. D. Kim, and H. S. Kang, "Activation of heat shock factor 1 by pyrrolidine dithiocarbamate is mediated by its activities as pro-oxidant and thiol modulator," Biochemical and Biophysical Research Communications, vol. 281, no. 2, pp. 367-372, 2001. 
[150] J. S. Thompson, R. Asmis, J. Glass et al., "P53 status influences regulation of HSPs and ribosomal proteins by PDTC and radiation," Biochemical and Biophysical Research Communications, vol. 343, no. 2, pp. 435-442, 2006.

[151] L. Klaic, R. I. Morimoto, and R. B. Silverman, "Celastrol analogues as inducers of the heat shock response. Design and synthesis of affinity probes for the identification of protein targets," ACS Chemical Biology, vol. 7, no. 5, pp. 928-937, 2012.

[152] B. T. Zhu and A. H. Conney, "Functional role of estrogen metabolism in target cells: review and perspectives," Carcinogenesis, vol. 19, no. 1, pp. 1-27, 1998.

[153] L. Klaic, P. C. Trippier, R. K. Mishra, R. I. Morimoto, and R. B. Silverman, "Remarkable stereospecific conjugate additions to the Hsp90 inhibitor celastrol," Journal of the American Chemical Society, vol. 133, no. 49, pp. 19634-19637.

[154] B. Peng, L. Xu, F. Cao et al., "HSP90 inhibitor, celastrol, arrests human monocytic leukemia cell U937 at G0/G1 in thiolcontaining agents reversible way," Molecular Cancer, vol. 9, article 79, 2010.

[155] A. T. Dinkova-Kostova, C. Abeygunawardana, and P. Talalay, "Chemoprotective properties of phenylpropenoids, bis(benzylidene)cycloalkanones, and related Michael reaction acceptors: correlation of potencies as phase 2 enzyme inducers and radical scavengers," Journal of Medicinal Chemistry, vol. 41, no. 26, pp. 5287-5296, 1998.

[156] S. R. Spencer, L. Xue, E. M. Klenz, and P. Talalay, “The potency of inducers of $\mathrm{NAD}(\mathrm{P}) \mathrm{H}$ :(quinone-acceptor) oxidoreductase parallels their effiency as substrates for glutathione transferases. Structural and electronic correlations," Biochemical Journal, vol. 273, no. 3, pp. 711-717, 1991.

[157] A. Rossi, G. Elia, and M. G. Santoro, "2-Cyclopenten-1one, a new inducer of heat shock protein 70 with antiviral activity," Journal of Biological Chemistry, vol. 271, no. 50, pp. 32192-32196, 1996.

[158] G. Elia, B. Polla, A. Rossi, and M. G. Santoro, "Induction of ferritin and heat shock proteins by prostaglandin A1 in human monocytes. Evidence for transcriptional and posttranscriptional regulation," European Journal of Biochemistry, vol. 264, no. 3, pp. 736-745, 1999.

[159] G. H. Snyder, M. J. Cennerazzo, A. J. Karalis, and D. Field, "Electrostatic influence of local cysteine environments on disulfide exchange kinetics," Biochemistry, vol. 20, no. 23, pp. 6509-6519, 1981.

[160] F. Hong, M. L. Freeman, and D. C. Liebler, "Identification of sensor cysteines in human Keap1 modified by the cancer chemopreventive agent sulforaphane," Chemical Research in Toxicology, vol. 18, no. 12, pp. 1917-1926, 2005.

[161] C. Hu, A. L. Eggler, A. D. Mesecar, and R. B. Van Breemen, "Modification of Keap1 cysteine residues by sulforaphane," Chemical Research in Toxicology, vol. 24, no. 4, pp. 515-521, 2011.

[162] N. Wakabayashi, A. T. Dinkova-Kostova, W. D. Holtzclaw et al., "Protection against electrophile and oxidant stress by induction of the phase 2 response: fate of cysteines of the Keap1 sensor modified by inducers," Proceedings of the National Academy of Sciences of the United States of America, vol. 101, no. 7, pp. 2040-2045, 2004.

[163] T. Yamamoto, T. Suzuki, A. Kobayashi et al., "Physiological significance of reactive cysteine residues of Keap1 in determining Nrf2 activity," Molecular and Cellular Biology, vol. 28, no. 8, pp. 2758-2770, 2008.
[164] N. A. Kaidery, R. Banerjee, L. Yang et al., “Targeting Nrf2mediated gene transcription by extremely potent synthetic triterpenoids attenuate dopaminergic neurotoxicity in the MPTP mouse model of Parkinson's disease," Antioxidants \& Redox Signaling, vol. 18, no. 2, pp. 139-157, 2013.

[165] A. L. Eggler, E. Small, M. Hannink, and A. D. Mesecar, "Cul3-mediated Nrf2 ubiquitination and antioxidant response element (ARE) activation are dependent on the partial molar volume at position 151 of Keap1," Biochemical Journal, vol. 422, no. 1, pp. 171-180, 2009.

[166] D. J. Manalo and A. Y. C. Liu, "Resolution, Detection, and Characterization of Redox Conformers of Human HSF1," Journal of Biological Chemistry, vol. 276, no. 26, pp. 23554-23561, 2001.

[167] L. Whitesell and S. L. Lindquist, "HSP90 and the chaperoning of cancer," Nature Reviews Cancer, vol. 5, no. 10, pp. 761-772, 2005.

[168] S. Tsutsumi, M. Mollapour, C. Prodromou et al., "Charged linker sequence modulates eukaryotic heat shock protein 90 (Hsp90) chaperone activity," Proceedings of the National Academy of Sciences of the United States of America, vol. 109, no. 8, pp. 2937-2942, 2012.

[169] S. Chen, W. P. Sullivan, D. O. Toft, and D. F. Smith, "Differential interactions of p23 and the TPR-containing proteins Hop, Cyp40, FKBP52 and FKBP51 with Hsp90 mutants," Cell Stress \& Chaperones, vol. 3, no. 2, pp. 118-129, 1998.

[170] J. C. Young, W. M. J. Obermann, and F. U. Hartl, "Specific binding of tetratricopeptide repeat proteins to the C-terminal $12-\mathrm{kDa}$ domain of hsp90," Journal of Biological Chemistry, vol. 273, no. 29, pp. 18007-18010, 1998.

[171] B. T. Scroggins, K. Robzyk, D. Wang et al., "An Acetylation Site in the Middle Domain of Hsp90 Regulates Chaperone Function," Molecular Cell, vol. 25, no. 1, pp. 151-159, 2007.

[172] M. Mollapour, S. Tsutsumi, A. C. Donnelly et al., "Swe1Wee1Dependent Tyrosine Phosphorylation of Hsp90 Regulates Distinct Facets of Chaperone Function," Molecular Cell, vol. 37, no. 3, pp. 333-343, 2010.

[173] S. K. Wandinger, M. H. Suhre, H. Wegele, and J. Buchner, "The phosphatase Ppt1 is a dedicated regulator of the molecular chaperone Hsp90," EMBO Journal, vol. 25, no. 2, pp. 367-376, 2006.

[174] I. Jorge, E. M. Casas, M. Villar et al., "High-sensitivity analysis of specific peptides in complex samples by selected MS/MS ion monitoring and linear ion trap mass spectrometry: application to biological studies," Journal of Mass Spectrometry, vol. 42, no. 11, pp. 1391-1403, 2007.

[175] K. Y. Rhee, H. Erdjument-Bromage, P. Tempst, and C. F. Nathan, "S-nitroso proteome of Mycobacterium tuberculosis: enzymes of intermediary metabolism and antioxidant defense," Proceedings of the National Academy of Sciences of the United States of America, vol. 102, no. 2, pp. 467-472, 2005.

[176] Y. Zhang, A. Keszler, K. A. Broniowska, and N. Hogg, "Characterization and application of the biotin-switch assay for the identification of S-nitrosated proteins," Free Radical Biology and Medicine, vol. 38, no. 7, pp. 874-881, 2005.

[177] A. Martínez-Ruiz, L. Villanueva, C. G. De Orduña et al., "Snitrosylation of Hsp90 promotes the inhibition of its ATPase and endothelial nitric oxide synthase regulatory activities," Proceedings of the National Academy of Sciences of the United States of America, vol. 102, no. 24, pp. 8525-8530, 2005.

[178] G. Nardai, B. Sass, J. Eber, G. Orosz, and P. Csermely, "Reactive cysteines of the 90-kDa heat shock protein, Hsp90," Archives of Biochemistry and Biophysics, vol. 384, no. 1, pp. 59-67, 2000. 
[179] M. Retzlaff, M. Stahl, H. C. Eberl et al., "Hsp90 is regulated by a switch point in the C-terminal domain," EMBO Reports, vol. 10, no. 10, pp. 1147-1153, 2009.

[180] G. Morra, G. Verkhivker, and G. Colombo, "Modeling signal propagation mechanisms and ligand-based conformational dynamics of the Hsp90 molecular chaperone full-length dimer," PLoS Computational Biology, vol. 5, no. 3, Article ID e1000323, 2009.

[181] D. L. Carbone, J. A. Doorn, Z. Kiebler, B. R. Ickes, and D. R. Petersen, "Modification of heat shock protein 90 by 4-hydroxynonenal in a rat model of chronic alcoholic liver disease," The Journal of Pharmacology and Experimental Therapeutics, vol. 315, no. 1, pp. 8-15, 2005.

[182] T. Shibata, Y. Kimura, A. Mukai et al., "Transthiocarbamoylation of proteins by thiolated isothiocyanates," The Journal of Biological Vhemistry, vol. 286, no. 49, pp. 42150-42161, 2011.

[183] A. T. Jacobs and L. J. Marnett, "Systems analysis of protein modification and cellular responses induced by electrophile stress," Accounts of Chemical Research, vol. 43, no. 5, pp. 673-683, 2010.

[184] A. Vila, K. A. Tallman, A. T. Jacobs, D. C. Liebler, N. A. Porter, and L. J. Marnett, "Identification of protein targets of 4hydroxynonenal using click chemistry for ex vivo biotinylation of azido and alkynyl derivatives," Chemical Research in Toxicology, vol. 21, no. 2, pp. 432-444, 2008.

[185] R. L. Smathers, J. J. Galligan, B. J. Stewart, and D. R. Petersen, "Overview of lipid peroxidation products and hepatic protein modification in alcoholic liver disease," Chemico-Biological Interactions, vol. 192, no. 1-2, pp. 107-112, 2011.

[186] Y. Li, G. E. Karagoz, Y. H. Seo et al., "Sulforaphane inhibits pancreatic cancer through disrupting Hsp90-p50(Cdc37) complex and direct interactions with amino acids residues of Hsp90," The Journal of Nutritional Biochemistry, vol. 23, no. 12, pp. 1617-1626, 2012.

[187] R. E. Connor, L. J. Marnett, and D. C. Liebler, "Proteinselective capture to analyze electrophile adduction of Hsp90 by 4-hydroxynonenal," Chemical Research in Toxicology, vol. 24, no. 8, pp. 1275-1282, 2011.

[188] Q. Liu, E. J. Levy, and W. J. Chirico, "N-ethylmaleimide inactivates a nucleotide-free Hsp70 molecular chaperone," Journal of Biological Chemistry, vol. 271, no. 47, pp. 29937-29944, 1996.

[189] W. J. Chirico, M. L. Markey, and A. L. Fink, "Conformational changes of an Hsp70 molecular chaperone induced by nucleotides, polypeptides, and N-ethylmaleimide," Biochemistry, vol. 37, no. 39, pp. 13862-13870, 1998.

[190] A. Hermawan and W. J. Chirico, "N-ethylmaleimide-modified Hsp70 inhibits protein folding," Archives of Biochemistry and Biophysics, vol. 369, no. 1, pp. 157-162, 1999.

[191] Y. Miyata, J. N. Rauch, U. K. Jinwal et al., "Cysteine reactivity distinguishes redox sensing by the heat-inducible and constitutive forms of heat shock protein 70," Chemistry \& Biology, vol. 19, no. 11, pp. 1391-1399, 2012.

[192] G. Siligardi, B. Panaretou, P. Meyerc et al., "Regulation of Hsp90 ATPase activity by the Co-chaperone Cdc37p/p50cdc37," Journal of Biological Chemistry, vol. 277, no. 23, pp. 20151-20159, 2002.

[193] S. M. Roe, M. M. U. Ali, P. Meyer et al., "The Mechanism of Hsp90 Regulation by the Protein Kinase-Specific Cochaperone p50cdc37," Cell, vol. 116, no. 1, pp. 87-98, 2004.

[194] S. Sreeramulu, S. L. Gande, M. Göbel, and H. Schwalbe, "Molecular mechanism of inhibition of the human protein complex Hsp90-Cdc37, a kinome chaperone-cochaperone, by triterpene celastrol," Angewandte Chemie, vol. 48, no. 32, pp. 5853-5855, 2009.

[195] M. D. Maines and J. F. Ewing, "Stress response of the rat testis: in situ hybridization and immunohistochemical analysis of heme oxygenase-1 (HSP32) induction by hyperthermia," Biology of Reproduction, vol. 54, no. 5, pp. 1070-1079, 1996.

[196] J. F. Ewing, V. S. Raju, and M. D. Maines, "Induction of heart heme oxygenase-1 (HSP32) by hyperthermia: possible role in stress-mediated elevation of cyclic $3^{\prime}: 5^{\prime}$-guanosine monophosphate," The Journal of Pharmacology and Experimental Therapeutics, vol. 271, no. 1, pp. 408-414, 1994.

[197] V. S. Raju and M. D. Maines, "Coordinated expression and mechanism of induction of HSP32 (heme oxygenase-1) mRNA by hyperthermia in rat organs," Biochimica et Biophysica Acta, vol. 1217, no. 3, pp. 273-280, 1994.

[198] J. F. Ewing, S. N. Haber, and M. D. Maines, "Normal and heatinduced patterns of expression of heme oxygenase-1 (HSP32) in rat brainhyperthermia causes rapid induction of mRNA and protein," Journal of Neurochemistry, vol. 58, no. 3, pp. 1140-1149, 1992.

[199] J. F. Ewing and M. D. Maines, "Rapid induction of heme oxygenase $1 \mathrm{mRNA}$ and protein by hyperthermia in rat brain: heme oxygenase 2 is not a heat shock protein," Proceedings of the National Academy of Sciences of the United States of America, vol. 88, no. 12, pp. 5364-5368, 1991.

[200] S. K. Niture and A. K. Jaiswal, "Hsp90 interaction with INrf2(Keap1) mediates stress-induced Nrf2 activation," Journal of Biological Chemistry, vol. 285, no. 47, pp. 36865-36875, 2010. 

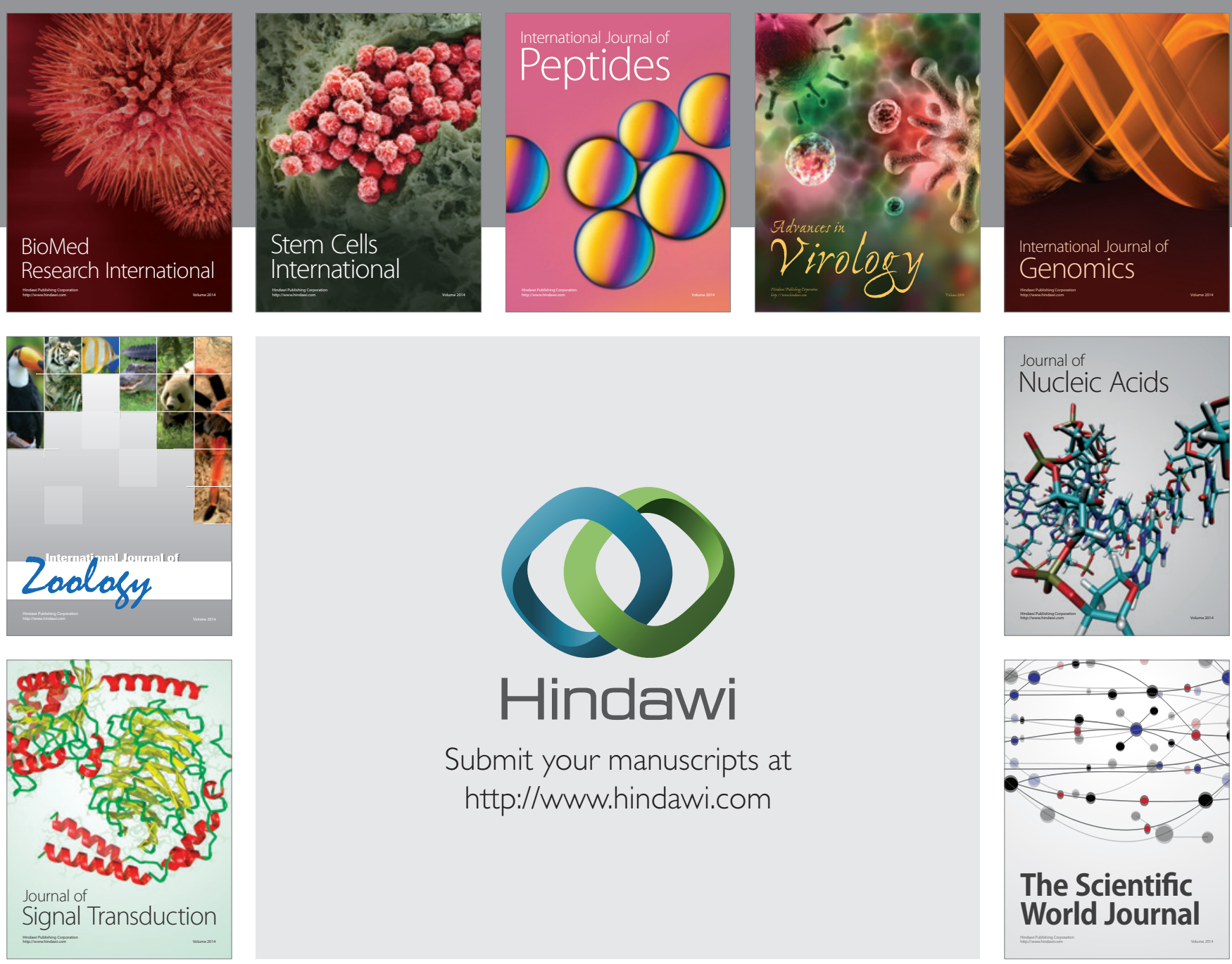

Submit your manuscripts at

http://www.hindawi.com
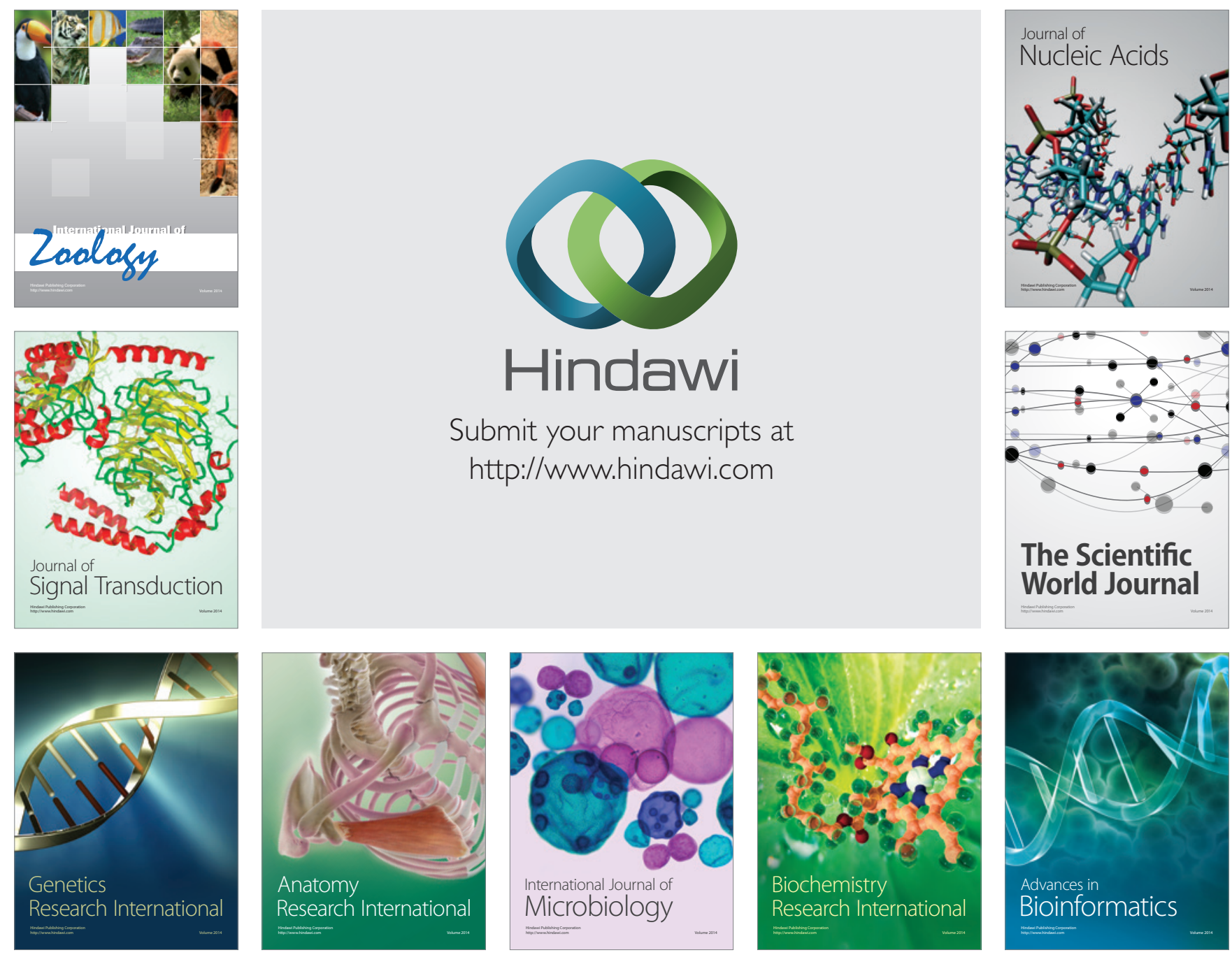

The Scientific World Journal
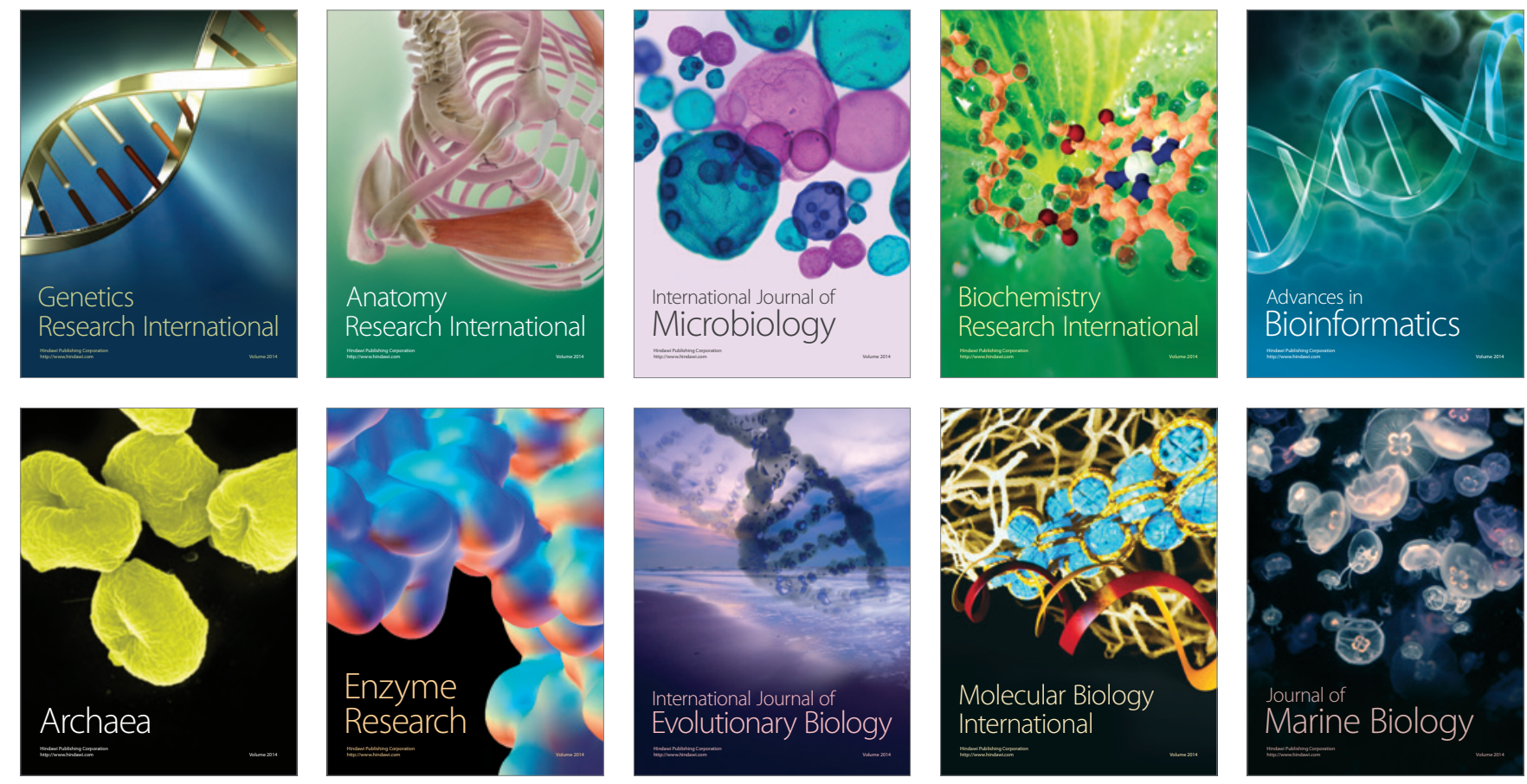